\title{
Der Übergang von der Grundschule in die weiterführende Schule: \\ Die Rolle von Schüler- und Klassenmerk- malen beim Einschätzen der individuellen Lernkompetenz durch die Lehrkräfte
}

\section{Kai Maaz, Marko Neumann, Ulrich Trautwein, Wolfgang Wendt, Rainer Lehmann und Jürgen Baumert}

Der Übergang in die verschiedenen Bildungsgänge des Sekundarschulsystems ist eine zentrale Statuspassage im Leben junger Menschen. Der vorliegende Beitrag untersucht die Lernkompetenzeinschätzungen von Lehrkräften am Ende der Grundschule. Neben dem prädiktiven Effekt individueller Leistungsmerkmale sowie von Indikatoren des sozialen Hintergrunds wurde untersucht, ob die Lernkompetenzeinschätzung der Lehrkräfte in einem systematischen Zusammenhang mit der mittleren Leistungsstärke einer Klasse steht. Die empirischen Analysen basieren aufeiner Stichprobe von 976 Schülerinnen und Schülern am Ende der Grundschulzeit in Berlin. In Mehrebenenanalysen waren Leistungsindikatoren sowie der sozioökonomische Status erwartungsgemäss positiv mit der Lernkompetenzeinschätzung assoziiert. Darüber hinaus fand sich bei Kontrolle der individuellen Leistung ein negativer Regressionskoeffizient des mittleren Leistungsniveaus der Klasse auf die Lernkompetenzeinschätzung, der als Referenzgruppeneffekt interpretiert werden kann.

\section{Einleitung}

Ein zentrales Strukturmerkmal des deutschen Bildungssystems ist die frühe Leistungsdifferenzierung, die sich in den unterschiedlichen Schulformen bzw. Bildungsgängen des Sekundarschulsystems ausdrückt. Während nur in Berlin und in Brandenburg sowie demnächst in Hamburg die Grundschulzeit sechs Jahre umfasst, erfolgt die Differenzierung in den anderen Ländern bereits nach der vierten Klassenstufe, zuweilen abgemildert durch Orientierungsstufen (z.B. Mecklenburg-Vorpommern). Ähnliche Formen der Differenzierung finden sich auch in den anderen deutschsprachigen Staaten (Maaz, Neumann \& Trautwein, in Druck). Die Leistungsdifferenzierung geht einher mit dem Erwerb unterschiedlicher Abschlusszertifikate, die wiederum die nachfolgenden Ausbildungsoptionen beeinflussen und damit auch berufliche Karrieren bahnen. Der Wechsel von der Grundschule in die jeweilige weiterführende Schule ist ohne Zweifel eine der zentralen Statuspassagen im Leben junger Menschen (Arnold, Bos, Richert \& Stubbe, 2007; Maaz, Hausen, McElvany \& Baumert, 2006). 
Die durch die Leistungsdifferenzierung entstehende Homogenisierung soll es erleichtern, alle Schülerinnen und Schüler bestmöglich zu fördern. In den vergangenen Jahren ist das gegliederte Schulsystem wieder verstärkt in die Kritik geraten. Eine Reihe von Kritikern stellt das Prinzip der Leistungsdifferenzierung generell infrage (Heinzel \& Prengel, 2002; Rösner, 2007). Andere wollen die Leistungsdifferenzierung beibehalten, verweisen aber auf Mängel beim Zuweisungsprozess auf eine der unterschiedlichen Leistungsgruppen. Oftmals wird in Hinblick auf die verwendeten Übertrittsverfahren von einer frühzeitigen Vergabe von Lebenschancen auf Basis kritikwürdiger Verfahren gesprochen (Baeriswyl, Trautwein, Wandeler \& Lüdtke, 2007; Ingenkamp \& Lissmann, 2005; Rösner, 2007). Schulleistungsstudien zeigen in der Tat, dass die Bewertungsstandards über verschiedene Klassen und Schülergruppen hinweg nicht einheitlich sind, was dazu führt, dass sich die Leistungsverteilungen an den verschiedenen Schulformen in der Sekundarstufe substanziell überlappen (Baumert, Trautwein \& Artelt, 2003).

Grundschulempfehlungen basieren in den meisten Ländern in erster Linie auf Schulnoten. In einer Reihe von Ländern gibt es ein weiteres Element der Empfehlung dadurch, dass die Klassenlehrerinnen und -lehrer gehalten sind, die Lernkompetenz ihrer Schülerinnen und Schüler einzuschätzen, also zum Beispiel deren kognitive Leistungsfähigkeit, ihr Arbeitsverhalten und ihre Lernmotivation. Die ergänzende Beurteilung soll angesichts der bekannten Grenzen der Notenvergabe dazu beitragen, den Übergangsprozess gerechter zu gestalten. Allerdings fehlt es bislang an Studien, die einen genaueren Einblick in die Güte sowie Bedingungsfaktoren dieser zusätzlichen Komponente erlauben. Sind Lernkompetenzeinschätzungen weniger anfällig als Noten gegenüber unerwünschten Effekten des sozialen Hintergrunds sowie der Klassenzusammensetzung? Anhand einer Stichprobe aus Berlin, wo die Lernkompetenzeinschätzungen eine kritische Bedeutung beim Übergang haben, untersucht der Beitrag die Rolle familiärer und kontextueller Determinanten von Schulnoten sowie der ergänzenden Kompetenzeinschätzungen.

\section{Theoretischer Hintergrund}

\section{Schulnoten im Rahmen des übergangsprozesses}

Das deutsche Bildungssystem ist stark föderal geprägt. Die einzelnen Bundesländer unterscheiden sich nicht nur in Hinblick auf die Zahl der angebotenen Bildungsgänge im Sekundarschulsystem, sie unterscheiden sich teilweise auch recht deutlich in den konkreten Übergangsregelungen (KMK, 2006), zum Beispiel beim Entscheidungsspielraum der Eltern. Unterschiede gibt es ebenfalls in der Art und Weise, wie die Leistungsfähigkeit der Schülerinnen und Schüler festgestellt wird, etwa durch Einsatz standardisierter Leistungstests oder durch das Erstellen von ausführlichen Grundschulgutachten, die Aspekte wie das Arbeits- 
verhalten oder die Motivation berücksichtigen (Ditton $\&$ Krüsken, 2006). In allen Ländern jedoch kommt den Schulnoten für die Bildungsgangempfehlung eine starke Bedeutung zu. So fanden Bos et al. (2004; Arnold et al., 2007) im Rahmen der Internationalen Grundschul-Lese-Untersuchung IGLU einen besonders engen Zusammenhang zwischen Schulnoten und Schullaufbahnempfehlungen, wobei zugleich die Leistungen in standardisierten Tests substanziell mit Noten und der Schullaufbahnempfehlung assoziiert waren.

Befunde zur Reliabilität und Validität von Schulnoten (z.B. Baumert et al., 2003) weisen allerdings ebenfalls darauf hin, dass der Zusammenhang zwischen Schulnoten und Testleistungen keineswegs perfekt ist, sondern teilweise durch den Einfluss von individuellen Schülermerkmalen wie Geschlecht und soziale Herkunft überlagert wird. Ferner ist zu berücksichtigen, dass die Basis von Noten und die Basis von Leistungstests nicht deckungsgleich sind. Noten dienen der Leistungsbewertung von Schülerinnen und Schülern. Eine Schulnote, wie sie beispielsweise in einem Zeugnis dokumentiert wird, setzt sich aus verschiedenen Einzelleistungen der Schülerinnen und Schüler zusammen. Hierzu zählen neben schriftlichen Arbeiten (z.B. Klassenarbeiten) auch mündliche Beiträge der Schülerinnen und Schüler im Unterricht und die Sorgfalt bei den Hausaufgaben. Greift man bei der Vorhersage von Schulnoten ausschliesslich auf standardisierte Leistungstests zurück, kann nur eine Komponente - die schriftliche Leistung der Schülerin bzw. des Schülers -, aus der sich eine Schulnote zusammen gesetzt, berücksichtigt werden. Der in vielen Studien nachgewiesene Effekt der sozialen Herkunft auf die Notenvergabe könnte demnach eine Folge einer Unterspezifikation des Modells sein.

Darüber hinaus ist bereits aus früheren Studien bekannt, dass bei der Notenvergabe keine klassenübergreifenden Standards greifen (Ingenkamp, 1993). Lehrkräfte orientieren sich vorwiegend am mittleren Leistungsniveau der jeweiligen Klasse. Der klasseninterne Bezugsrahmen führt dazu, dass Bewertungsmassstäbe über verschiedene Klassen hinweg unterschiedlich sind. Somit gibt es Klassen, in denen die mittlere Note identisch ausfällt, deren anhand von standardisierten Tests ermittelten Leistungen jedoch deutlich differieren.

\section{Referenzgruppeneffekte im übergangsprozess}

Die zuletzt beschriebenen Befunde (Bos et al., 2004; Ingenkamp, 1993) sowie die starke Bedeutung der Noten für die Übertrittsempfehlungen lassen es möglich erscheinen, dass bei der Einschätzung der Lernkompetenz systematische Referenzgruppeneffekte zwischen einzelnen Klassen zum Tragen kommen. Solche Referenzgruppeneffekte sind in der pädagogischen Psychologie ein gut bekanntes Phänomen. Besonderes Forschungsinteresse fanden in den vergangenen Jahren Referenzgruppeneffekte auf das schulische Selbstkonzept, die umfassend und auf methodisch hohem Niveau untersucht wurden (Marsh, 2005). Besonders prominent ist hier der sogenannte Big-Fish-Little-Pond-Effekt, der sich darin äussert, dass Schülerinnen und Schüler mit vergleichbaren Leistungen in Ab- 
hängigkeit der mittleren Leistungsstärke der Klasse unterschiedliche Einschätzungen ihrer eigenen fachlichen Fähigkeiten entwickeln. Je höher das Leistungsniveau in der Klasse, umso niedriger die Einschätzung des fachbezogenen Selbstkonzepts.

In der jüngeren Vergangenheit konnte gezeigt werden, dass sich Referenzgruppeneffekte nicht auf das Selbstkonzept beschränken. Trautwein, Gerlach und Lüdtke (in Druck) fanden Referenzgruppeneffekte der mittleren motorischen Leistungsfähigkeit der Schulklasse auf sportliche Aktivitäten in Sportvereinen. Bei gleichen individuellen motorischen Leistungen berichteten Schülerinnen und Schüler aus Klassen, in denen die mittlere motorische Leistungsfähigkeit hoch lag, im Mittel über weniger sportliche Aktivitäten in Sportvereinen; dieser Effekt war teilweise über das sportbezogene Selbstkonzept vermittelt.

Marsh (1987), Trautwein, Lüdtke, Marsh, Köller \& Baumert (2006) und Trautwein et al. (in Druck) konnten darüber hinaus zeigen, dass sich die Referenzgruppeneffekte nicht auf Schülervariablen beschränken; vielmehr besteht auch ein systematischer Zusammenhang zwischen der mittleren, mit einem standardisierten Leistungstest ermittelten Leistungsstärke von Klassen bzw. Schulen und den in diesen Klassen bzw. Schulen erteilten Schulnoten: Je stärker die Mitschülerinnen und Mitschüler, desto schlechter fällt - bei gleicher Testleistung die erhaltene Schulnote aus.

Bislang liegen nur wenige empirische Arbeiten vor, die die Auswirkungen von Referenzgruppeneffekten auf den Übergangsprozess untersucht haben. Die Befundlage der vorliegenden Studien ist jedoch eindeutig. Bei Kontrolle des individuellen Leistungsstandes fallen die Übergangsempfehlungen in leistungsstarken Klassen weniger positiv aus als in weniger leistungsstarken Klassen (Tiedemann \& Billmann-Mahecha, 2007; Trautwein \& Baeriswyl, 2007).

\section{Die Rolle der sozialen Herkunft im übergangsprozess}

Die Befunde der ersten PISA-Studie haben das Problem sozialer Disparitäten im Bildungssystem wieder in den Fokus der Öffentlichkeit gerückt. Baumert und Schümer (2001) ermittelten mit den Daten der PISA-2000-Studie zwischen den Sozialschichten variierende Zugangschancen zu den verschiedenen Bildungsgängen des Sekundarschulsystems. Ihre Analysen ergaben, dass die Chance für den Gymnasialbesuch bei Kindern aus Oberschichtfamilien sehr viel höher war als bei Kindern aus weniger begünstigten Familien - auch bei Kontrolle der tatsächlichen Leistungen und der kognitiven Grundfähigkeit. In der Folge wurde dieser Effekt in verschiedenen Studien repliziert (u.a. Ditton, 2007; Ditton \& Krüsken, 2006; Merkens \& Wessel, 2002).

Diese Befunde lassen sich auch in anderen, ähnlich strukturierten Bildungssystemen nachweisen (vgl. für die Schweiz: Ramseier \& Brühwiler, 2003; für Österreich: Bacher, 2003, 2005). Moser und Rhyn (2000) konnten für die Schweiz im Rahmen einer Untersuchung des Züricher Übertrittsverfahrens von 
der Primarstufe in die Sekundarstufe I einen ähnlichen sozial selektiven Effekt bei der Empfehlung für einen anspruchsvolleren Schultyp nachweisen. Baeriswyl, Wandeler, Trautwein und Oswald (2006) untersuchten das Übertrittsverfahren an Deutschfreiburger Schulen, das durch die Verwendung von Leistungstests sowie einer obligatorischen Beratung der Eltern Effekte des familiären Hintergrunds gering halten soll. Wenngleich der soziale Hintergrund eine relativ geringe Prädiktionskraft aufwies, war er trotzdem statistisch signifikant.

Der sozial selektive Effekt bei der Übergangsempfehlung bedarf jedoch einer gewissen Relativierung. Ditton (2005) stellte in einer bayerischen Stichprobe die Bildungswünsche der Eltern den Grundschulempfehlungen der Lehrkräfte gegenüber. Bei vergleichbaren Leistungen der Kinder waren die relativen Chancen, dass Eltern, die selbst das Abitur erworben haben, für ihre Kinder das Gymnasium anstelle einer anderen Schulform wählten, 8,84-mal so gross wie die von Eltern, die maximal einen Hauptschulabschluss aufwiesen. Für die Grundschulempfehlung berichtete Ditton dagegen einen deutlich geringeren Effekt (odds ratio $=3,92$ ). Vergleicht man die Ergebnisse mit der Bildungsaspiration der Eltern, dann ist zu erkennen, dass die Empfehlungen der Lehrkräfte weniger an die soziale Herkunft gekoppelt sind als die Bildungsaspirationen der Eltern. Diesen Ergebnissen zufolge wirkt die Grundschulempfehlung also «sozial korrigierend» und sozial selektiv zugleich.

\section{Institutionelle Rahmung des übergangsprozesses am Beispiel Berlins}

Da die institutionelle Ausgestaltung des Übergangsprozesses von Bundesland zu Bundesland verschieden ist, variiert dementsprechend die Bedeutung der einzelnen Elemente, zum Beispiel die Verbindlichkeit der Grundschulempfehlung und Ausmass der elterlichen Entscheidungsfreiheit. Analysen zum Übergang in die Sekundarstufe I müssen deshalb der institutionellen Variabilität zwischen den Bundesländern Rechnung tragen. Die vorliegende Studie nimmt den Übergang am Ende der Grundschule in Berlin in den Blick. Der Übergang in die weiterführenden Bildungsgänge des Sekundarschulsystems erfolgt in Berlin nach der sechsten Klassenstufe. Bereits Anfang der 1950er Jahren wurde im Berliner Schulgesetz festgeschrieben, dass für jedes Kind am Ende der sechsten Klassenstufe ein ausführliches Gutachten anzufertigen sei, das mit einer Empfehlung für einen der drei Zweige der Oberschule endet. Mitte der 1950er Jahre kam es zu einer deutlichen Stärkung des Elternwunsches (Thiel, 2005). Heute ist der Übergang in die Sekundarstufe I in Berlin durch den freien Elternwillen gekennzeichnet (Berliner SchulG $\$ 56$, Absatz (3)). Voraussetzung für die Wahl eines Bildungsganges ist jedoch die Eignung für diesen Bildungsgang, die von der Grundschule in einer Bildungsgangempfehlung ${ }^{1}$ festgestellt wird, ohne allerdings bindenden Charakter zu haben.

Die Bildungsgangempfehlung wird in Klassenstufe 6 frühestens drei Wochen vor Ausgabe der Halbjahreszeugnisse erstellt und beschlossen. Darin wird ein 
Bildungsgang (Hauptschule, Realschule oder Gymnasium) empfohlen, der für die weitere Entwicklung des Kindes am geeignetsten erscheint. Grundlage für die Empfehlung sind die erzielten Leistungen (Fachnoten) und die weiteren Lernkompetenzeinschätzungen: "Aus den Zeugnisnoten der Jahrgangsstufen 5 und 6 - bei doppelter Gewichtung der Noten in Jahrgangsstufe 6 - wird eine Durchschnittsnote gebildet; dabei werden die Fächer Deutsch, Fremdsprache, Mathematik und Naturwissenschaften verstärkt (Faktor 2) berücksichtigt. Bis zu einer Durchschnittsnote von einschliesslich 2,2 ist eine Gymnasialempfehlung, von 2,8 bis 3,2 eine Realschulempfehlung und ab 3,8 eine Hauptschulempfehlung zu erteilen. In den Zwischenbereichen (2,3 bis 2,7 und 3,3 bis 3,7) ist für die Empfehlung des Bildungsgangs die Einschätzung der Merkmale massgebend, die die Lernkompetenz kennzeichnen» (Grundschulverordnung, 2005, $\$$ 24, Absatz 2).

Die Lehrkräfte müssen für alle Schülerinnen und Schüler deren Lernkompetenz einschätzen. Diese Einschätzung ist für die Empfehlungsvergabe in ganz bestimmten Fällen massgebend. Bislang ist nur sehr wenig darüber bekannt, wie die Einschätzung erfolgt und welche Faktoren den Prozess beeinflussen.

\section{Fragestellung}

Die vorliegende Studie berücksichtigt individuelle Schülermerkmale sowie die leistungsbezogene und soziale Komposition der Schulklassen bei der Vorhersage von Schulnoten und Lernkompetenzeinschätzungen durch die Lehrkräfte am Ende der Grundschulzeit. Im Mittelpunkt stehen drei Fragestellungen.

\section{Bedeutung der Lernkompetenzeinschätzung in der Bildungsgangempfehlung}

Die Lernkompetenzeinschätzungen der Grundschullehrkräfte im Rahmen des Übergangsprozesses am Ende der Grundschulzeit besitzen in Berlin unmittelbare Bedeutung, wenn auf Grundlage der Durchschnittsnote ${ }^{2}$ keine eindeutige Empfehlung ausgesprochen werden kann. In den oben beschriebenen Übergangsregelungen ist dieser Entscheidungsspielraum für die Alternativen Hauptschule vs. Realschule sowie Realschule vs. Gymnasium explizit vorgesehen. In diesem Zusammenhang interessieren deskriptiv drei Fragen: (1) Finden sich Abweichungen zwischen den in den Übergangsvorschriften festgesetzten Vorgaben und den erteilten Bildungsgangempfehlungen? Gibt es also Schülerinnen und Schüler, die auf Grundlage der Durchschnittsnote zum Beispiel eine Realschulempfehlung bekommen sollten, denen aber eine andere Empfehlung (Hauptschul- oder Gymnasialempfehlung) erteilt wurde? (2) Wie gross ist der Anteil der Schülerinnen und Schüler, für die die Lehrkräfte einen individuellen Entscheidungsspielraum haben und für die die vorzunehmende Lernkompetenzeinschätzung damit unmittelbar relevant wird? (3) Sofern Lehrkräfte bei der Empfehlungsvergabe nicht an die Durchschnittsnote der Schülerinnen und Schüler gebunden sind, wählen sie dann häufiger die Empfehlung für den anspruchsvolleren oder den weniger anspruchsvolleren Bildungsgang? 


\section{Determinanten der Schulnoten}

Die erzielten Noten nehmen entscheidenden Einfluss auf die Bildungsgangempfehlung. Wir erwarten analog zu den Befunden aus den grossen Schulleistungsstudien im Primarschulbereich (Arnold et al., 2007; Bos et al., 2004), dass es zwischen Noten und Leistungen aus standardisierten Tests einen positiven $\mathrm{Zu}-$ sammenhang gibt. Gleichzeitig wird davon ausgegangen, dass sich prädiktive Effekte des sozioökonomischen Status auf die Notenvergabe ermitteln lassen, wonach Kinder aus sozial begünstigten Familien bessere Noten erreichen als Kinder aus sozial weniger begünstigten Familien - auch dann, wenn die Testleistung kontrolliert wird. Darüber hinaus gibt es Hinweise, dass Mädchen besser benotet werden als Jungen. Ebenfalls in Anlehnung an jüngere Studien erwarten wir den Nachweis von Referenzgruppeneffekten bei der Notenvergabe. Die Analysen von Tiedemann und Billmann-Mahecha (2007) sowie von Trautwein und Baeriswyl (2007) haben gezeigt, dass sich Referenzgruppeneffekte bei der Vergabe der Empfehlung für einen weiterführenden Bildungsgang und den tatsächlich realisierten Übergang nachweisen lassen. Bei gleicher individueller Schulleistung - gemessen über einen standardisierten Leistungstest - sollten die Noten in leistungsstarken Klassen vergleichsweise schlechter ausfallen als in leistungsschwachen Klassen.

\section{Determinanten der Lernkompetenzeinschätzung durch die Lehrkräfte}

Wir gehen davon aus, dass der Einschätzung der Lernkompetenz ein ähnliches Zusammenhangsmuster zugrunde liegt, wie es bereits bei der Notenvergabe beschrieben wurde. Die Testleistungen sollten in einem positiven Zusammenhang mit der Lernkompetenzeinschätzung stehen. Mädchen sollten insgesamt besser eingeschätzt werden als Jungen und Kinder sozial begünstigter Familien besser als Kinder aus sozial weniger begünstigten Familien. Die Effekte des sozioökonomischen Status sollten sich deutlich reduzieren, wenn die Testleistung der Schülerinnen und Schüler sowie die Durchschnittsnote aus den Bildungsgangempfehlungen kontrolliert werden, wobei letztere den stärksten Zusammenhang mit der Lernkompetenzeinschätzung aufweisen dürften.

Von besonderer Wichtigkeit war uns darüber hinaus, potenzielle Referenzgruppeneffekte auf die Einschätzung der Lernkompetenz durch die Lehrkräfte zu untersuchen. Wir erwarten, dass die individuelle Leistung der Schülerinnen und Schüler in einem standardisierten Test die Einschätzung der Lernkompetenz durch die Lehrkräfte voraussagen würde: Je besser die Testleistung, desto grösser die geschätzte Lernkompetenz durch die Lehrkräfte. Gleichzeitig sollte jedoch auch ein negativer Effekt der mittleren Klassenleistung zu finden sein. Bei gleicher individueller Testleistung sollte ein Schüler oder eine Schülerin eine weniger günstige Lernkompetenzeinschätzung in denjenigen Klassen erhalten, in denen die mittlere Leistung der Mitschülerinnen und -schüler besonders gut ausfällt.

Zusätzlich zur Vorhersagekraft der mittleren Klassenleistung in Hinblick auf die Noten bzw. den Übergang untersuchten wir auch die Vorhersagekraft der so- 
zialen Komposition der Klasse. Da es an entsprechenden Vorgängerstudien mangelt, handelte es sich hierbei um eher explorative Analysen. Ein Blick in die Literatur zum Referenzgruppeneffekt (vgl. Abschnitt 2) und der in verschiedenen Studien nachgewiesene Zusammenhang zwischen Leistung der Schülerinnen und Schüler und der sozialen Herkunft einerseits und die Kovariation zwischen Empfehlung und Schülerleistung andererseits lassen auf einen möglichen Effekt der sozialen Komposition der Klassen schliessen. Es interessiert, ob sich auch für die soziale Zusammensetzung ein statistisch signifikanter Effekt finden lässt und in welche Richtung dieser weist.

\section{Methode}

\section{Stichprobe}

Die Daten der vorliegenden Studie entstammen der in Berlin durchgeführten Untersuchung «Erhebungen zum Lese- und Mathematikverständnis - Entwicklungen in den Jahrgangsstufen 4 bis 6 in Berlin» (ELEMENT). In dieser längsschnittlich angelegten Untersuchung wurde die Entwicklung der Deutsch- und Mathematikleistungen der Schülerinnen und Schüler des Primarbereichs von der vierten bis zur sechsten Klasse untersucht (Lehmann \& Lenkeit, 2008). In Jahrgangsstufe 6 wurden zudem die Englischleistungen querschnittlich erhoben. Die Leistungstests wurden durch Schüler-, Eltern- und Lehrerbefragungen ergänzt.

Anders als in den meisten Bundesländern wechselt der Grossteil der Schülerinnen und Schüler in Berlin erst nach der 6. Jahrgangsstufe in die weiterführenden Schulen der gegliederten Sekundarstufe I. Für besonders leistungsstarke Schülerinnen und Schüler besteht allerdings die Möglichkeit, bereits nach der vierten Klasse auf ein grundständiges Gymnasium zu wechseln. Für den Jahrgang der ELEMENT-Untersuchung lag der Anteil der vorzeitigen Übergänger an das Gymnasium bei rund sieben Prozent der Gesamtschülerschaft.

In der ELEMENT-Untersuchung wurde sowohl die Leistungsentwicklung der vorzeitigen Übergänger (Vollerhebung mit $N=1.724$ Schülerinnen und Schülern zur ersten Erhebungswelle) als auch der regulären Grundschülerschaft untersucht, für die ein repräsentatives Sample von 3.293 Schülerinnen und Schülern aus 71 Grundschulen mit 140 Klassen (Bruttostichprobe erste Erhebungswelle) gezogen wurde. Da im vorliegenden Beitrag nur der Übergang nach der sechsten Klasse in die weiterführenden Schulen untersucht wird, beziehen sich alle Auswertungen und Analysen ausschliesslich auf das Sample der regulären Grundschülerschaft zum dritten Erhebungszeitpunkt in der sechsten Klassenstufe $(N=3.008)$. Allerdings handelt es sich dabei nur um einen Teil der Grundschulstichprobe, denn erst eine Anschlussuntersuchung in Jahrgangsstufe 8 (ELEMENT 8), die unter Federführung des Max-Planck-Instituts für Bildungsforschung durchgeführt wurde, machte die im Zentrum der vorliegenden 
Untersuchung stehenden Lernkompetenzeinschätzungen aus den Bildungsgangsempfehlungen zugänglich und überführte sie in den Datensatz. Um die ELEMENT-Schülerinnen und -schüler in die Nachuntersuchung einbeziehen zu können, bedurfte es zunächst dreier zentraler Voraussetzungen. Zum einen mussten die Zuordnungslisten von Schülername und Schüler-ID, die aus datenschutzrechtlichen Gründen in den ehemaligen Grundschulen der Schülerinnen und Schüler verwahrt wurden, noch vorhanden sein, um die nacherhobenen Daten mit den bereits vorhandenen Schülerdaten verbinden zu können. Zum anderen musste herausgefunden werden, an welche Schulen die Schülerinnen und Schüler nach dem Verlassen der Grundschule gegangen waren, da die Bildungsgangempfehlungen samt Lernkompetenzeinschätzungen Bestandteil der Schülerakten sind, die von der Grundschule an die weiterführende Schule weitergegeben werden. Und schliesslich bedurfte es der Bereitschaft der Grund- und der weiterführenden Schulen, die verfügbaren Informationen bereitzustellen.

Letztlich lagen von insgesamt 976 Schülerinnen und Schülern aus 83 Klassen (50\% Mädchen) und damit von etwa 30 Prozent des ursprünglichen Grundschulsamples verwertbare Angaben aus den Bildungsgangempfehlungen vor. Da nicht ohne weiteres von einem zufälligen Ausfall von Grund- bzw. Sekundarschulen ausgegangen werden kann, stellt sich die Frage, wie repräsentativ die für unsere Analysen herangezogene Stichprobe ist. Wie den Tabellen 1a und b entnommen werden kann, unterscheidet sich die Gruppe der Schülerinnen und Schüler mit vorhandenen Lernkompetenzeinschätzungen nur verhältnismässig gering von der Gruppe ohne vorliegende Lernkompetenzeinschätzungen, auch wenn die vorhandenen Unterschiede ausnahmslos statistisch signifikant ausfielen. Vergleicht man die Teilgruppe der Schülerinnen und Schüler mit vorhandenen Lernkompetenzeinschätzungen mit der gesamten Grundschulstichprobe, reduzieren sich die Unterschiede nochmals. Hinsichtlich der Leistungsindikatoren für die sechste Klasse (Leistungstests und Noten) fallen die Unterschiede gegenüber dem Gesamtsample bis auf den Mathematiktest $(d=0,14)$ geringer als ein zehntel Standardabweichung aus. Dasselbe gilt für den sozioökonomischen Status, für den sich ebenfalls leicht höhere Werte als im Gesamtsample finden. Die in Tabelle 1b aufgeführten prozentualen Angaben zur beruflichen Ausbildung der Eltern, dem Migrationsstatus und der ausgesprochenen Übergangsempfehlung machen ebenfalls deutlich, dass bei der von uns herangezogenen Teilstichprobe erwartungsgemäss nicht von einem repräsentativen Sample Berliner Schülerinnen und Schüler ausgegangen werden kann, wenngleich die Unterschiede zwischen den «Teilnehmern» und «Nicht-Teilnehmern» relativ gering ausfielen. 
Tabelle 1a: Mittelwerte und Standardabweichungen zentraler Leistungsindikatoren und des sozioökonomischen Status für die Gruppen mit und ohne Bildungsgangempfehlung und die Gesamtstichprobe

\begin{tabular}{|c|c|c|c|c|c|c|}
\hline & \multicolumn{2}{|c|}{$\begin{array}{l}\text { Bildungsgangempfeh- } \\
\text { lung liegt vor } \\
\text { (Analysestichprobe, } \\
\mathrm{N}=976 \text { ) }\end{array}$} & \multicolumn{2}{|c|}{$\begin{array}{l}\text { Bildungsgangempfeh- } \\
\text { lung liegt nicht vor } \\
\quad(\mathrm{N}=2.032)\end{array}$} & \multicolumn{2}{|c|}{$\begin{array}{l}\text { Gesamtstichprobe } \\
(\mathrm{N}=3.008)\end{array}$} \\
\hline & M & SD & M & SD & M & SD \\
\hline Lesetest & 110,82 & 13,2 & 109,44 & 12,27 & 109,93 & 12,52 \\
\hline Mathematiktest & 116,87 & 15,6 & 113,91 & 14,86 & 114,86 & 15,18 \\
\hline Kognitive Grundfähigkeit ${ }^{1}$ ) & 26,38 & 10,09 & 25,28 & 9,87 & 25,64 & 10,00 \\
\hline Deutschnote & 2,75 & 0,94 & 2,85 & 0,88 & 2,82 & 0,90 \\
\hline Mathematiknote & 2,91 & 1,11 & 3,02 & 1,05 & 2,99 & 1,05 \\
\hline Fremdsprachnote & 2,93 & 1,03 & 3,02 & 1,11 & 2,98 & 1,11 \\
\hline ISEI ${ }^{2)}$ & 47,89 & 16,28 & 45,80 & 15,38 & 46,50 & 15,69 \\
\hline
\end{tabular}

Anmerkungen: $\mathrm{M}=$ Mittelwert; $\mathrm{SD}=$ Standardabweichung

1) Kognitive Grundfähigkeit $=$ Kognitiver Fähigkeitstest, KFT.

2) ISEI = International Socio-Economic Index of Occupational Status, (Sozioökonomischer Status, höchster Wert in der Familie).

Tabelle 1b: Prozentuale Verteilung zentraler Hintergrundindikatoren und der vergebenen Übergangsempfehlung für die Gruppen mit und ohne Bildungsgangempfehlung und die Gesamtstichprobe

\begin{tabular}{|lccc|}
\hline & $\begin{array}{c}\text { Bildungsgangemp- } \\
\text { fehlung liegt vor } \\
\text { Analysestichprobe, } \\
\mathrm{N}=976)\end{array}$ & $\begin{array}{c}\text { Bildungsgangempfeh- } \\
\text { lung liegt nicht vor }\end{array}$ & Gesamtstichprobe \\
\hline Berufliche Ausbildung & & & \\
- ohne Abschluss & $11,8 \%$ & $13,6 \%$ & $(\mathrm{~N}=3.008)$ \\
- Lehre & $27,6 \%$ & $25,8 \%$ & $13,0 \%$ \\
- Fachschulabschluss & $29,4 \%$ & $31,5 \%$ & $26,4 \%$ \\
- FH-Abschluss & $11,7 \%$ & $10,7 \%$ & $30,8 \%$ \\
- Uni-Abschluss & $19,6 \%$ & $18,4 \%$ & $11,0 \%$ \\
Migrationshintergrund & $13,2 \%$ & $12,1 \%$ & $18,8 \%$ \\
- ein Elternteil im Ausland geb. & $25,0 \%$ & $28,4 \%$ & $27,3 \%$ \\
- beide Elternteile im Ausland geb. & & & \\
Übergangsempfehlung & $18,2 \%$ & $19,2 \%$ & $18,9 \%$ \\
- Hauptschule & $40,4 \%$ & $46,0 \%$ & $44,1 \%$ \\
- Realschule & $41,4 \%$ & $34,8 \%$ & $37,0 \%$ \\
- Gymnasium & & & \\
\hline
\end{tabular}


Der Umgang mit fehlenden Werten stellt eine besondere Herausforderung in der empirischen Forschung dar. Tabelle 2 gibt einen Überblick der fehlenden Werte für die vorliegende Analysestichprobe. Wie Tabelle 2 entnommen werden kann, finden sich die meisten fehlenden Werte für die Elternangaben zur beruflichen Ausbildung, zum ausgeübten Beruf (ISEI) und zum Migrationshintergrund, während der Anteil für die übrigen Analysevariablen bis auf den Test zu den kognitiven Grundfähigkeiten (KFT) unter 10 Prozent beträgt. Da der Ausschluss von Personen mit fehlenden Werten die Validität der Befunde beeinträchtigen kann, setzt sich in der Forschung zunehmend die Position durch, fehlende Werte durch den Einsatz leistungsstarker Algorithmen zu schätzen anstatt einen fallbzw. listenweisen Ausschluss von Personen vorzunehmen (vgl. Allison, 2001; Lüdtke, Robitzsch, Trautwein \& Köller, 2007). In der vorliegenden Untersuchung wurden die fehlenden Werte nach dem Multiple-Imputation-Verfahren (Schafer \& Graham, 2002) mit Hilfe des Programms NORM 2.03 (Schafer, 1999) geschätzt. Dabei wurden neben den Analysevariablen auch eine Reihe weiterer sogenannter Hilfsvariablen, von denen angenommen wurde, dass sie in Beziehung zum Ausfallprozess stehen, für die Schätzung der fehlenden Werte herangezogen. Zu den Hilfsvariablen zählten unter anderem Skalen zum akademischen Selbstkonzept, zur Lernmotivation, zum Leseinteresse sowie das mittlere Leistungsniveau und die soziale Zusammensetzung der Schulklassen. Insgesamt wurden fünf vollständige Datensätze erzeugt, d.h. es wurden für jede Person fünf Werte in allen fehlenden Variablen geschätzt, die die Grundlage für die empirischen Analysen bildeten. Alle Auswertungen wurden fünfmal gerechnet und die Ergebnisse nach dem von Rubin (1987) vorgeschlagenen Vorgehen verbunden.

Tabelle 2: Übersicht über die fehlenden Werte in den Analysevariablen

\begin{tabular}{|lc|}
\hline & Anteil fehlender Werte in $\%$ \\
\hline Geschlecht & $0,0 \%$ \\
Berufliche Ausbildung & $29,1 \%$ \\
Migrationshintergrund & $29,2 \%$ \\
ISEI & $32,6 \%$ \\
Lesetest & $4,6 \%$ \\
Mathematiktest & $4,6 \%$ \\
Kognitive Grundfähigkeit & $12,3 \%$ \\
Deutschnote & $5,4 \%$ \\
Mathematiknote & $2,9 \%$ \\
Lernkompetenzeinschätzung * & $3,9-6,6 \%$ \\
Übergangsempfehlung & $0,0 \%$ \\
\hline
\end{tabular}

Anmerkung: ISEI = International Socio-Economic Index of Occupational Status,

(Sozioökonomischer Status, höchster Wert in der Familie)

* Range der fehlenden Werte auf den Einzelitems der Lernkompetenzeinschätzung. 


\section{Variablen und Instrumente}

Lernkompetenzeinschätzungen durch die Lehrkräfte. Im Rahmen der Bildungsgangempfehlung haben die Lehrkräfte die Lernkompetenz aller ihrer Schüler eingeschätzt. Insgesamt lagen den Lehrkräften 17 Statements zur Lernkompetenz der Schülerinnen und Schüler vor, die sie anhand einer vierstufigen Antwortskala einschätzen sollten $(1=$ nicht sehr ausgeprägt, $2=$ durchschnittlich ausgeprägt, 3 = gut ausgeprägt, 4 = besonders ausgeprägt). Beispielitems: «findet selbständig Lösungswege», «kann Konflikte konstruktiv lösen». Ob und wieweit die Auswahl der 17 Items theoriegeleitet erfolgte, liess sich nicht ermitteln. Jedenfalls sollten die Lehrkräfte keine spezifischen Einschätzungen ausgewählter Dimensionen, wie zum Beispiel Motivation oder kognitive Fähigkeiten, vornehmen. Inhaltlich lassen sich die Statements jedoch post-hoc unterschiedlichen Dimensionen zuordnen. (1) Fachwissen (7 Items), Beispielitem: «kennt Fakten, Begriffe und Definitionen und wendet sie sachgerecht an», (2) Regulation (4 Items), Beispielitem: «kann Konflikte konstruktiv lösen», (3) Arbeitstechniken (4 Items), Beispielitem: «kann Arbeitsschritte planen und organisieren» und (4) Offenheit und Fantasie (2 Items), Beispielitem: «ist Neuem gegenüber aufgeschlossen und vielseitig interessiert». Die letzte Dimension wurde in den vorliegenden Analysen ausgeschlossen, weil die entsprechenden Items eher die Persönlichkeit als die Lernkompetenz der Schülerinnen und Schüler messen, sodass 15 Items in die weitere Auswertung eingingen.

Da die Einschätzung der Lernkompetenz lediglich als Globalurteil eine praktische Bedeutung im Übergangsprozess hat und eine entsprechende Gesamtskala mit den 15 Items eine sehr gute interne Konsistenz aufwies (Cronbachs Alpha = .97), haben wir für die nachfolgend berichteten empirischen Analysen das Lehrkraftrating als Gesamtskala herangezogen. Für weitergehende Detailanalysen mag es hingegen teilweise sinnvoll sein, das Nested-Faktor-Modell als Ausgangspunkt der Analysen zu verwenden. ${ }^{3}$

Bildungshintergrund der Eltern. Aus den im Elternfragebogen gemachten Angaben zum Bildungshintergrund beider Elternteile wurde eine Variable mit dem jeweils höchsten Bildungsabschluss gebildet, die fünf Abschlussniveaus beinhaltet: (1) ohne Abschluss einer Berufsausbildung oder eines Studiums, (2) abgeschlossene Berufsausbildung, (3) Abschluss einer Fachschulausbildung, (4) Abschluss eines Fachhochschulstudiums, (5) Abschluss eines Universitätsstudiums.

Migrationshintergrund. Zur Erfassung des Migrationsstatus wurden die Eltern gefragt, in welchem Land sie selbst geboren sind. Aus diesen Angaben wurde ein Indikator mit folgenden Ausprägungen gebildet: $1=$ beide Eltern sind in Deutschland geboren; 2 = nur ein Elternteil ist in Deutschland geboren; $3=$ beide Eltern sind im Ausland geboren.

Sozioökonomische Stellung der Eltern. Zur Beschreibung der sozioökonomischen Stellung dient der auf den elterlichen Berufsangaben basierende Internationale Sozioökonomische Index (ISEI), den Ganzeboom, DeGraaf, Treiman \& De Leeuw (1992) entwickelt haben. Für die Analysen wurde der höchste sozioökonomische Index von Vater bzw. Mutter gewählt. 
Schulischer Leistungsindex. Zur Bestimmung der Leseleistungen am Ende der sechsten Klasse wurden Tests zum Leseverstehen eingesetzt, die bereits in der Hamburger Studie LAU für Klassenstufe 6 und 7 (Aspekte der Lernausgangslage und der Lernentwicklung; Lehmann, Gänsfuss \& Peek, 1999) sowie in der IGLU-Studie (Bos et al., 2003) zum Einsatz kamen. Der Test bestand aus vier kurzen Prosatexten und einem Gebrauchstext, zu denen anschliessend Fragen zum Verständnis gestellt wurden. Unter den insgesamt 37 Fragen befanden sich 6 Aufgaben mit offenem Antwortformat, die restlichen Fragen wurden im Multiple Choice-Format vorgelegt. Die Reliabilität des Tests war mit .84 (Kuder-Richardson Formula 20) zufriedenstellend. Die Mathematikleistung wurde mit Instrumenten aus der LAU-Untersuchung (Lehmann et al., 1999), der IGLU-Studie (Bos et al., 2003) sowie der Brandenburgischen Mathematikuntersuchung QuaSUM (vgl. Lehmann et al., 2001) gemessen. Die insgesamt 49 Aufgaben stammten zum grössten Teil aus den Stoffgebieten Arithmetik und Geometrie. Die Reliabilität des Tests fiel mit .92 (Kuder-Richardson Formula 20) gut aus. Beide Tests wurden unter Nutzung des Partial-Credit-Modells Rasch-skaliert. Bei den Personenparametern handelt es sich jeweils um gewichtete Likelihood-Schätzer (Warm, 1989). Um einen validen Leistungsindikator für Berücksichtigung der leistungsbezogenen Komposition der Klasse zu bekommen und der Multikollinearität auf Klassenebene zu begegnen, wurde aus den Testleistungen der Schülerinnen und Schüler in Lesen und Mathematik ein Leistungsindex gebildet, indem die beiden Leistungswerte nach einer Standardisierung an der Gesamtstichprobe $(N=3.008)$ gemittelt wurden.

Kognitive Grundfähigkeiten. Die kognitiven Grundfähigkeiten der Grundschüler wurden am Ende der vierten Klasse (erste Erhebungswelle) mit zwei Untertests des Kognitiven Fähigkeitstests (KFT, Heller \& Perleth, 2000) erhoben. Die Subtests erfassen verbales und figurales Schlussfolgern und gelten als Markertest für fluide Intelligenz. Die Reliabilität des Gesamttests betrug .93 (Kuder-Richardson Formula 20).

Durchschnittsnote. Als Indikator für die Noten wurde der in den Bildungsgangempfehlungen errechnete Notendurchschnitt verwendet. Dieser berücksichtigt Noten ab dem Schuljahr 5. Noten aus dem ersten Halbjahr der Klassenstufe 6 gehen doppelt in die Berechnung ein. Darüber hinaus werden die Mathematik-, Deutsch- sowie die Note der ersten Fremdsprache doppelt gewichtet. Der so errechnete Notendurchschnitt ist ausschlaggebend für die Empfehlungsvergabe (vgl. auch Abschnitt 2.4).

\section{Statistische Analysen}

In Untersuchungen mit Schulklassen ist die hierarchische Struktur der gewonnenen Daten zu berücksichtigen, um mögliche Fehlschlüsse bei der Analyse zu vermeiden (Raudenbush \& Bryk, 2002). Aufgrund der hierarchischen Datenstruktur sowie der simultanen Modellierung der Effekte individueller Merkmale (individuelle Testleistung) bzw. der Schulklasse (über die Schülerinnen und 
Schüler einer Klasse gemittelte Testleistung) verbietet sich die konventionelle Regressionsanalyse zur Hypothesenprüfung (Raudenbush \& Bryk, 2002). Ein adäquates Auswertungsverfahren für Daten mit hierarchischer Struktur stellt die Mehrebenenanalyse dar, eine spezielle Form der Regressionsanalyse, die es erlaubt, simultan Prädiktoren auf mehreren Ebenen (z. B. Individualebene, Klassenebene) zu berücksichtigen.

Die gängigen Softwarepakete für Mehrebenenanalysen wie das hier benutzte Programm HLM 6 (Raudenbush, Bryk, Cheong \& Congdon, 2004) bieten allein unstandardisierte Regressionskoeffizienten in den Ergebnisausdrucken an, was die Interpretation der Regressionsgewichte aufgrund der oftmals arbiträren Metriken der Prädiktoren und Kriterien erschwert. Um die spätere Interpretation zu vereinfachen, wurden alle nichtdichotomen Variablen $z$-standardisiert $(M=0 ; S D=1)$. Die resultierenden Koeffizienten können daher annähernd im Sinne standardisierter Regressionskoeffizienten interpretiert werden. Für die Vorhersage der Noten aus den Bildungsgangempfehlungen wurde die Note in ihrer ursprünglichen Metrik verwendet. Die Testleistung und der sozioökonomische Status wurden auf Klassenebene aggregiert und anschliessend erneut standardisiert. Für die Erzeugung der Kompositionsmerkmale wurden die Leistungswerte und sozialen Hintergrundmerkmale der gesamten Grundschulstichprobe berücksichtigt, um die soziale und leistungsbezogene Komposition der Schulklasse angemessen zu erfassen.

Um die Reliabilität der auf Klassenebene aggregierten Merkmale zu bestimmen, wurde die Intraklassenkorrelation (ICC) berechnet. Die Ausprägungen für die sogenannte ICC(1) zeigen, dass zwischen 25 Prozent (ISEI) und 34 Prozent (Leistungsindikator) der Varianz in den genannten Indikatoren zwischen den Klassen lagen. Aus der ICC(1) und der mittleren Klassen- bzw. Gruppengrösse lässt sich als Wert für die Reliabilität des Klassenmittelwertes der Kompositionsmerkmale die ICC(2) berechnen (Bliese, 2000; Lüdtke, Trautwein, Kunter \& Baumert, 2006; Snijders \& Bosker, 1999). Die ICC(2) kann als Genauigkeit der über alle Schülerinnen und Schüler einer Klasse gemittelten leistungsbezogenen und sozialen Kompositionsmerkmale interpretiert werden. Die im vorliegenden Fall errechnete ICC(2) spricht insgesamt für eine sehr gute Reliabilität der auf Klassenebene gemittelten Leistungsindikatoren $(0,92)$ und des sozioökonomischen Status $(0,88)$.

\section{Ergebnisse}

\section{Empfehlungsvergabe und Deskriptive Analysen}

Wenngleich die Grundschulempfehlung für einen weiterführenden Bildungsgang in Berlin keinen bindenden Charakter hat, folgt die Vergabe der Empfehlung selbst klaren Notenvorgaben. Betrachtet man die tatsächlich vergebene Empfehlung am Ende der Grundschule mit den Empfehlungen, die auf Grund- 
lage des in der Bildungsgangempfehlung ermittelten Notendurchschnitts vergeben werden sollen, fallen Unterschiede auf. Diese Unterschiede lassen sich aber grösstenteils durch den in den Übergangsregelungen vorgesehenen Entscheidungsspielraum der Lehrkräfte erklären. Eine Übereinstimmung zwischen vergebener Empfehlung und der Empfehlungsvorgabe konnte insgesamt für 62 Prozent festgestellt werden, in 37 Prozent ist aufgrund des Notendurchschnitts in der Bildungsgangempfehlung kein Bildungsgang direkt zu empfehlen. Die Lehrerinnen und Lehrer sollen gerade in diesen Fällen für die Vergabe der Empfehlung die Lernkompetenzeinschätzungen der Schülerinnen und Schüler berücksichtigen. In weniger als ein Prozent $(0,6 \%)$ weicht die vergebene Empfehlung von der Vorgabe ab. Dies betrifft Einzelfälle, in denen Lehrkräfte zum Beispiel keine Gymnasialempfehlung ausgesprochen haben, obwohl die betroffenen Schülerinnen und Schüler aufgrund ihres Notendurchschnitts in der Bildungsgangempfehlung diese Empfehlung bekommen sollten.

\section{Tabelle 3: Verteilung der Grundschulempfehlungen am Ende der Grundschulzeit in Abhängigkeit der erwarteten Verteilung nach den Übergangsvorschriften (in Spalte und Zeilenprozent)}

\begin{tabular}{|lcccccc|}
\hline & \multicolumn{3}{c|}{ Verteilung der tatsächlich vergebenen Grundschulempfehlungen } \\
\hline Erwartete & \multicolumn{3}{c}{ Hauptschule $(18,4)$} & \multicolumn{2}{c|}{ Realschule $(40,1)$} & \multicolumn{2}{c|}{ Gymnasium (41,5) } \\
EmpfehlungI) & Spalte & Zeile & Spalte & Zeile & Spalte & Zeile \\
\hline Hauptschule (6,3) & 33,9 & 100 & -- & -- & -- & -- \\
Realschule (19,2) & $\mathbf{1 , 1}$ & 1,1 & $\mathbf{4 7 , 3}$ & 98,9 & -- & -- \\
Gymnasium (37,4) & -- & -- & $\mathbf{1 , 0}$ & 1,1 & $\mathbf{8 9 , 1}$ & 98,9 \\
E1 (21,5) & $\mathbf{0 , 6}$ & 0,5 & $\mathbf{4 2 , 2}$ & 78,6 & $\mathbf{1 0 , 9}$ & 21,0 \\
E2 (15,7) & $\mathbf{6 4 , 4}$ & 75,8 & $\mathbf{9 , 5}$ & 24,2 & -- & 0,0 \\
\hline
\end{tabular}

Anmerkungen: Fett gedruckte Zahlen stellen Spaltenprozente und kursiv gedruckte Zahlen Zeilenprozente dar.

E1 (Entscheidungsspielraum 1) = aus dem Notendurchschnitt in der Bildungsgangempfehlung folgt keine Grundschulempfehlung. Lehrkraft soll zwischen Realschul- und Gymnasialempfehlung entscheiden; E2 (Entscheidungsspielraum 2) = aus dem Notendurchschnitt in der Bildungsgangempfehlung folgt keine Grundschulempfehlung. Lehrkraft soll zwischen Haupt- und Realschulempfehlung entscheiden; in Klammern ausgewiesene Prozentangaben geben die jeweiligen Randverteilungen.

1) Empfehlung, die auf Grundlage der Noten nach der Berliner Grundschulverordnung vergeben werden sollte.

Die hohe Übereinstimmung lässt sich auch für die Empfehlungsvergabe für die verschiedenen Schulformen beobachten (vgl. Tab. 3). Von den Schülerinnen und Schülern, die aufgrund ihrer Noten eine Empfehlung für die Hauptschule zu erwarten hatten, bekamen alle diese Empfehlungen. Bei der Realschule und dem Gymnasium liegen die Übereinstimmungswerte bei jeweils 98,9 Prozent. In den Fällen, in denen die Lehrkräfte keine Empfehlung aufgrund des Noten- 
durchschnitts vergeben, folgen die Empfehlungen auch einem regelhaften Muster. So entschieden sich Lehrerinnen und Lehrer mehrheitlich (mehr als drei Viertel) für die jeweils niedrigere Schulform. Sollen die Lehrer beispielsweise zwischen Realschule und Gymnasium entscheiden, wählten 78,6 Prozent die Realschule und nur 21 Prozent das Gymnasium. Für die Entscheidungsalternative zwischen Realschule und Hauptschule konnte dieselbe Tendenz ermittelt werden.

Mittelwerte, Standardabweichungen sowie die Interkorrelationen zwischen den Untersuchungsvariablen sind in Tabelle 4 berichtet. Sofern es sich um mehrkategoriale Variablen handelt (trichotom und mehr), sind die Mittelwerte und Standardabweichungen in Abbildung 1 dargestellt. Die Lerneinschätzungen durch die Lehrkräfte liegen mit einem Mittelwert von $M=2,43$ und einer Standardabweichung von $S D=0,75$ geringfügig unter dem Mittelpunkt der Skala. Erwartungsgemäss fanden sich hohe bis sehr hohe Zusammenhänge zwischen der Lerneinschätzung und den Leistungsindikatoren. Der engste Zusammenhang resultierte mit $r=-.86$ zwischen der Lerneinschätzung der Lehrkräfte und dem Notendurchschnitt aus der Bildungsgangempfehlung.

\section{Tabelle 4: Deskriptive Befunde: Mittelwerte, Standardabweichungen und Korrela- tionen}

Anmerkung: $M=$ Mittelwert; $S D=$ Standardabweichung; Fett gedruckte Korrelationen sind statistisch si-

\begin{tabular}{|lrrrrrrr|}
\hline & $M$ & $S D$ & $(1)$ & $(2)$ & $(3)$ & $(4)$ & $(5)$ \\
\hline (1) ISEI & 47,89 & 16,28 & & & & & \\
(2) Mathematikleistung & 116,86 & 15,60 & $\mathbf{0 , 3 7}$ & & & & \\
(3) Leseleistung & 110,85 & 13,21 & $\mathbf{0 , 4 1}$ & $\mathbf{0 , 6 9}$ & & & \\
(4) Kognitive Grundfähigkeit & 26,38 & 10,09 & $\mathbf{0 , 3 4}$ & $\mathbf{0 , 5 9}$ & $\mathbf{0 , 5 6}$ & & \\
$\begin{array}{l}\text { (5) Notendurchschnitt } \\
\text { (Bildungsgangempfehlung) }\end{array}$ & 2,60 & 0,73 & $\mathbf{- 0 , 3 6}$ & $-\mathbf{0 , 6 2}$ & $\mathbf{- 0 , 6 1}$ & $\mathbf{- 0 , 5 0}$ & \\
$\begin{array}{l}\text { (6) Lerneinschätzungen } \\
\text { (Lehrerurteil) }\end{array}$ & 2,43 & 0,75 & $\mathbf{0 , 3 5}$ & $\mathbf{0 , 5 7}$ & $\mathbf{0 , 5 6}$ & $\mathbf{0 , 4 4}$ & $\mathbf{- 0 , 8 6}$ \\
\hline
\end{tabular}

gnifikant bei $\mathrm{p}<.05$; ISEI = International Socio-Economic Index of Occupational Status, (Sozioökonomischer Status, höchster Wert in der Familie).

Wie erwartet, fiel auch der Zusammenhang zwischen sozioökonomischem Status (ISEI) und den Leistungsindikatoren sowie den Lernkompetenzeinschätzungen positiv aus. Mit dem Bildungsniveau und dem Migrationsstatus konnte neben dem sozioökonomischen Status auch für weitere familiäre Hintergrundmerkmale ein statistisch signifikanter Zusammenhang mit der Lernkompetenzeinschätzung ermittelt werden. Kindern aus sozial begünstigten Familien werden demnach positivere Lernkompetenzeigenschaften zugeschrieben als Kindern aus weniger sozial begünstigten Familien. Darüber hinaus fallen 
die Lernkompetenzeinschätzungen bei den Mädchen positiver aus als bei den Jungen.

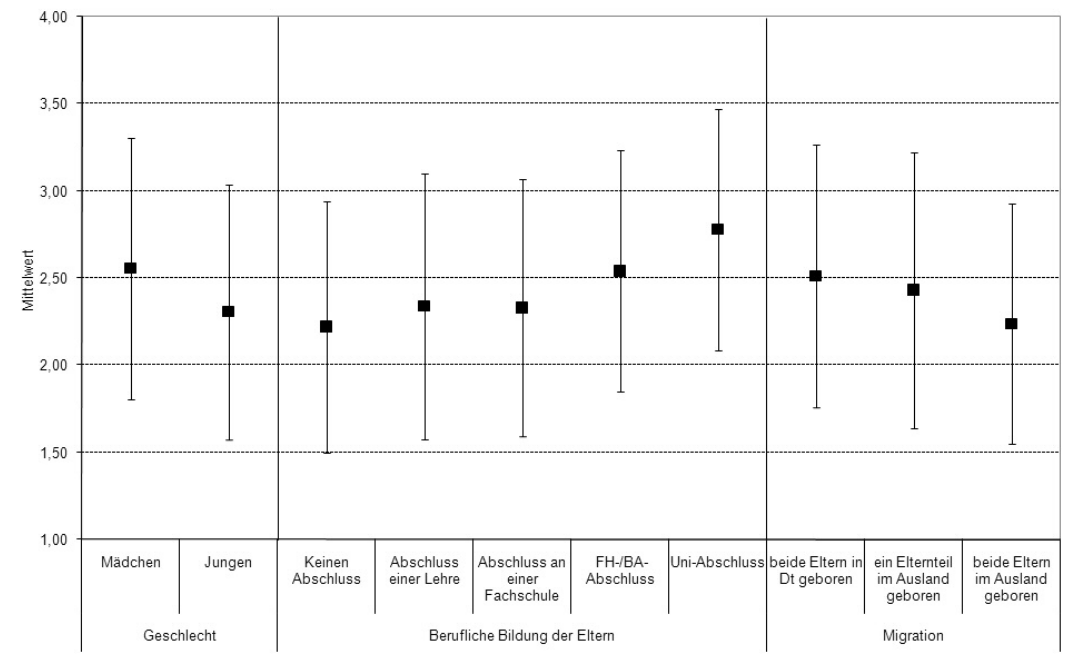

Anmerkung: Die kleinen fett gedruckten Quadrate bilden den Mittelwert ab, die vertikalen Linien beschreiben den Bereich, in dem rund zwei Drittel der jeweiligen Gruppe liegen (eine Standardabweichung unterhalb und oberhalb des Mittelwerts).

Abbildung 1: Deskriptive Befunde: Mittelwerte und Standardabweichungen der Lernkompetenzeinschätzung (Gesamtskala) differenziert nach Geschlecht, Bildungshintergrund und Migrationsstatus der Eltern

\section{Determinanten der Notenvergabe}

Zur Vorhersage der Schulnoten der Schülerinnen und Schüler, operationalisiert mit dem Notendurchschnitt in der Bildungsgangempfehlung, wurden eine Reihe von Mehrebenenmodellen spezifiziert, die in Tabelle 5 berichtet werden. Die Note wurde für diese Analysen nicht reckodiert. Somit steht ein hoher Wert für eine schlechte Note und ein niedriger Wert für eine gute. Betrachtet man zunächst die Individualebene (Modell 1 und 2), so zeigen sich die postulierten Effekte. Entsprechend unseren Erwartungen hatte die individuelle Leistung der standardisierten Tests einen positiven Effekt auf die Notenvergabe. Ebenfalls bestätigte sich der Geschlechtereffekt, wonach Mädchen in der Leistungsbewertung besser abschneiden als Jungen - auch dann, wenn die individuelle Testleistung kontrolliert wird. Ein deutlicher Effekt konnte auch für den sozioökonomischen Status ermittelt werden. Kinder aus sozial begünstigten Familien erhielten bessere Noten als Kinder aus sozial weniger begünstigten Familien. Bei Hinzunahme der individuellen Testleistungen in das Regressionsmodell verringert sich der Effekt des sozioökonomischen Status von $b=-0,18$ auf $b=-0,06$, erreichte aber weiterhin das Signifikanzkriterium von $p<0,05$. 
Im Einklang mit unseren Erwartungen konnte für die mittlere Klassenleistung $(b=0,11$; Modell 3) ein statistischer signifikanter Effekt auf die individuelle Durchschnittsnote ermittelt werden. Bei gleicher individueller Testleistung fiel die Benotung der Schülerinnen und Schüler in leistungsstarken Klassen weniger gut aus. Zudem zeigte sich auch für die soziale Zusammensetzung ( $b=0,09$; Modell 4) ein statistisch signifikantes Regressionsgewicht. Bei vergleichbarer sozialer Herkunft der Schülerinnen und Schüler fiel die Benotung der Schülerinnen und Schüler in sozial günstig zusammengesetzten Klassen weniger gut aus. Die simultane Berücksichtigung der leistungsbezogenen und sozialen Komposition (Modell 5) verdeutlicht die starke Konfundierung von leistungsbezogener und sozialer Zusammensetzung der Klasse. Die Effekte gehen jeweils zurück. Der spezifische Effekt der mittleren Klassenleistung $(b=0,11)$ erreicht nur noch auf dem 10-Prozent-Niveau $(p=0,057)$ statistische Signifikanz.

\section{Determinanten der Lernkompetenzbeurteilung}

Für die Analyse der Lernkompetenzeinschätzungen haben wir eine analoge Serie von Regressionsmodellen spezifiziert und im Rahmen von Mehrebenenanalysen getestet. Auf Individualebene haben wir neben den zentralen Indikatoren der sozialen Herkunft schrittweise die Indikatoren für die Leistung der Schülerinnen und Schüler in das Modell aufgenommen. Modell 1 (vgl. Tab. 6a) berücksichtigt ausschliesslich Hintergrundmerkmale. Das Geschlecht und der sozioökonomische Status weisen einen positiven prädiktiven Effekt auf. Die Lernkompetenzeinschätzungen fielen für Mädchen positiver aus als für Jungen, und Kinder aus sozial begünstigten Familien wurden besser eingeschätzt als Kinder aus weniger sozial begünstigten Familien. Darüber hinaus wurde ein signifikanter Effekt des elterlichen Bildungshintergrundes nachgewiesen. Kontrolliert man die individuelle Testleistung, die die grösste prädiktive Wirkung besitzt (Modell 2), verringert sich der SES-Effekt, erreicht aber weiterhin das Signifikanzkriterium von $p<0,05$. Im Modell 2a wurde zusätzlich die Durchschnittsnote aus der Bildungsgangempfehlung mit in das Modell aufgenommen, die einen spezifischen Effekt auf die Lernkompetenzeinschätzung hat. Von den Leistungsindikatoren erreicht nur noch der Leistungsindex das Signifikanzkriterium. Der Effekt des sozioökonomischen Status verringert sich weiter und ist nicht mehr signifikant. Auch das Geschlecht hat nach Kontrolle der Noten keinen eigenständigen Effekt mehr auf Lernkompetenzeinschätzung. Die Varianzaufklärung fiel mit 83 Prozent sehr hoch aus. Der Migrationshintergrund der Kinder stand in keinem negativen Zusammenhang mit den Lernkompetenzeinschätzungen der Lehrkräfte. Nach Kontrolle der Noten wies der Migrationshintergrund einen positiven Effekt auf die Lernkompetenzeinschätzungen auf. 


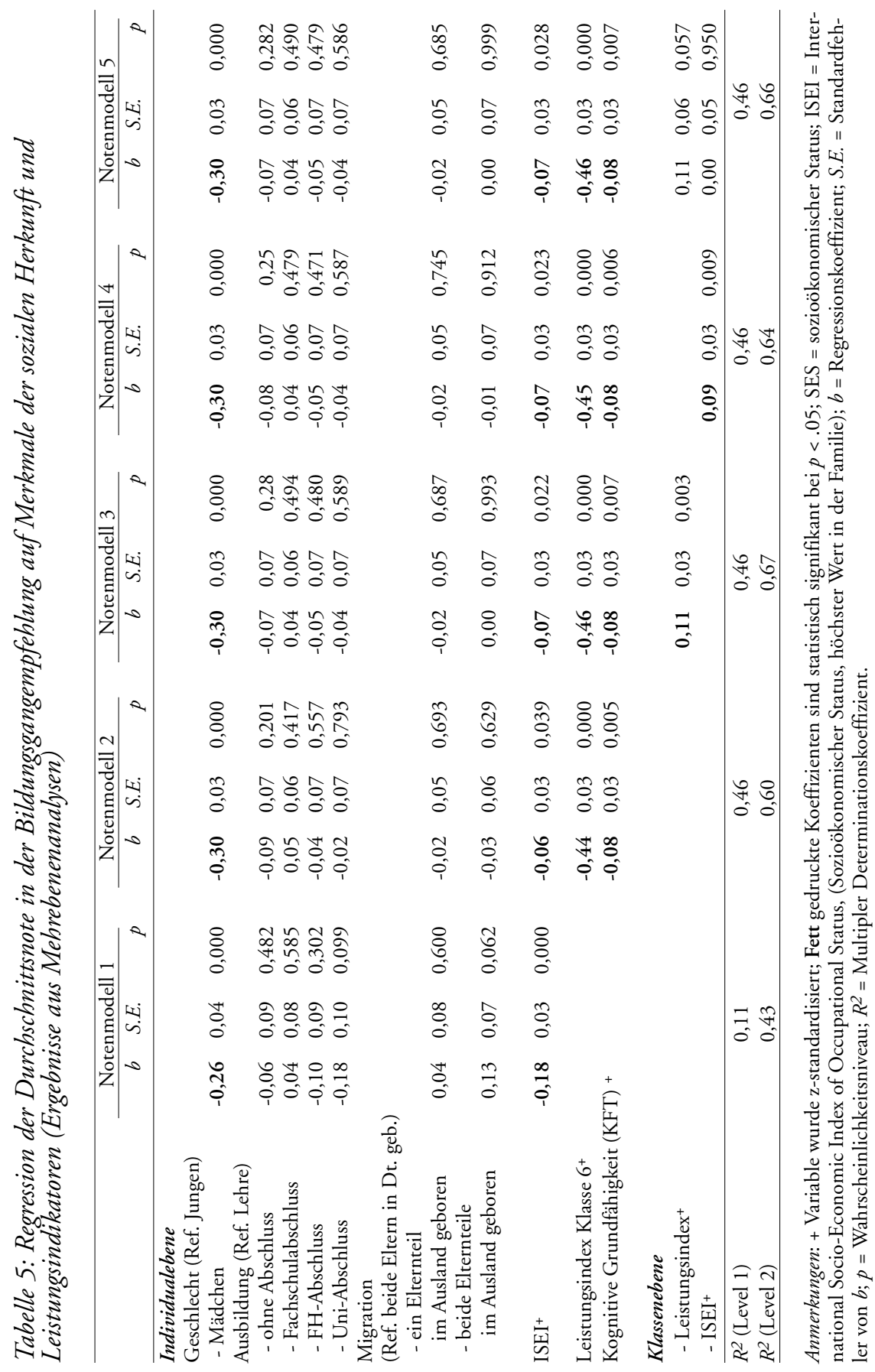


Tabelle 6a: Regression der Lernkompetenzeinschätzung durch die Lehrkräfte auf Merkmale der sozialen Herkunft und Leistungsindikatoren (Ergebnisse aus Mehrebenenanalysen)

\begin{tabular}{|c|c|c|c|c|c|c|c|c|c|}
\hline & \multicolumn{3}{|c|}{ Modell 1} & \multicolumn{3}{|c|}{ Modell 2} & \multicolumn{3}{|c|}{ Modell 2a } \\
\hline & $b$ & S.E. & $p$ & $b$ & S.E. & $p$ & $b$ & S.E. & $p$ \\
\hline \multicolumn{10}{|l|}{ Individualebene } \\
\hline \multicolumn{10}{|l|}{ Geschlecht (Ref. Jungen) } \\
\hline - Mädchen & 0,33 & 0,06 & 0,000 & 0,37 & 0,05 & 0,000 & 0,03 & 0,03 & 0,387 \\
\hline \multicolumn{10}{|l|}{ Ausbildung (Ref. Lehre) } \\
\hline - ohne Abschluss & 0,09 & 0,12 & 0,450 & 0,12 & 0,09 & 0,200 & 0,01 & 0,05 & 0,817 \\
\hline - Fachschulabschluss & $-0,05$ & 0,10 & 0,608 & $-0,06$ & 0,08 & 0,476 & 0,00 & 0,04 & 0,938 \\
\hline - FH-Abschluss & 0,14 & 0,12 & 0,227 & 0,07 & 0,09 & 0,473 & 0,02 & 0,05 & 0,688 \\
\hline - Uni-Abschluss & 0,27 & 0,12 & 0,025 & 0,06 & 0,08 & 0,494 & 0,03 & 0,06 & 0,579 \\
\hline \\
\hline \multirow{2}{*}{\multicolumn{10}{|c|}{$\begin{array}{l}\text { (Ref. beide Eltern in Dt. geb.) } \\
\text { - ein Elternteil }\end{array}$}} \\
\hline & & & & & & & & & \\
\hline \multicolumn{10}{|l|}{ - beide Elternteile } \\
\hline im Ausland geboren & $-0,05$ & 0,09 & 0,549 & 0,14 & 0,08 & 0,077 & 0,11 & 0,04 & 0,003 \\
\hline ISEI $^{+}$ & 0,25 & 0,04 & 0,000 & 0,10 & 0,03 & 0,006 & 0,02 & 0,02 & 0,253 \\
\hline \multicolumn{4}{|l|}{ Leistungsindex Klasse $6^{+}$} & 0,60 & 0,04 & 0,000 & 0,09 & 0,03 & 0,001 \\
\hline \multicolumn{4}{|c|}{ Kognitive Grundfähigkeit (KFT) + } & 0,08 & 0,04 & 0,058 & $-0,02$ & 0,02 & 0,408 \\
\hline \multicolumn{7}{|c|}{ Noten in der Bildungsgangempfehlung } & $-0,86$ & 0,03 & 0,000 \\
\hline$R^{2}$ (Level 1) & & 0,10 & & & 0,44 & & & 0,83 & \\
\hline$R^{2}($ Level 2) & & 0,37 & & & 0,35 & & & 0,58 & \\
\hline
\end{tabular}

Anmerkungen: + Variable wurde z-standardisiert; Fett gedruckte Koeffizienten sind statistisch signifikant bei $p<.05$; SES = sozioökonomischer Status; ISEI = International Socio-Economic Index of Occupational Status, (Sozioökonomischer Status, höchster Wert in der Familie); $b=$ Regressionskoeffizient; S.E. $=$ Standardfehler von $b ; p=$ Wahrscheinlichkeitsniveau; $R^{2}=$ Multipler Determinationskoeffizient.

Im Modell 3 wird die mittlere Klassenleistung in das Modell aufgenommen. Das Ergebnismuster auf Individualebene ist mit dem aus Modell 2 vergleichbar. Entsprechend unseren Erwartungen hatte die individuelle Testleistung einen positiven Effekt auf die Lernkompetenzeinschätzung. Darüber hinaus wurden signifikante Effekte des Geschlechts und des sozioökonomischen Status nachgewiesen. Die mittlere Klassenleistung stand in einem negativen Zusammenhang mit der Lernkompetenzbeurteilung $(b=-0,19)$ (vgl. Tab. 6b). Bei gleicher individueller Leistung fiel die Lernkompetenzbeurteilung in leistungsstarken Klassen weniger positiv aus.

In einem nächsten Schritt wurde der auf Klassenebene gemittelte sozioökonomische Hintergrund als Prädiktorvariable eingeführt. So lässt sich prüfen, ob - bei Kontrolle der übrigen Prädiktoren - Schülerinnen und Schüler in Klassen, die eine günstige soziale Zusammensetzung aufweisen, eher eine positive Lernkompetenzbeurteilung erhalten. 


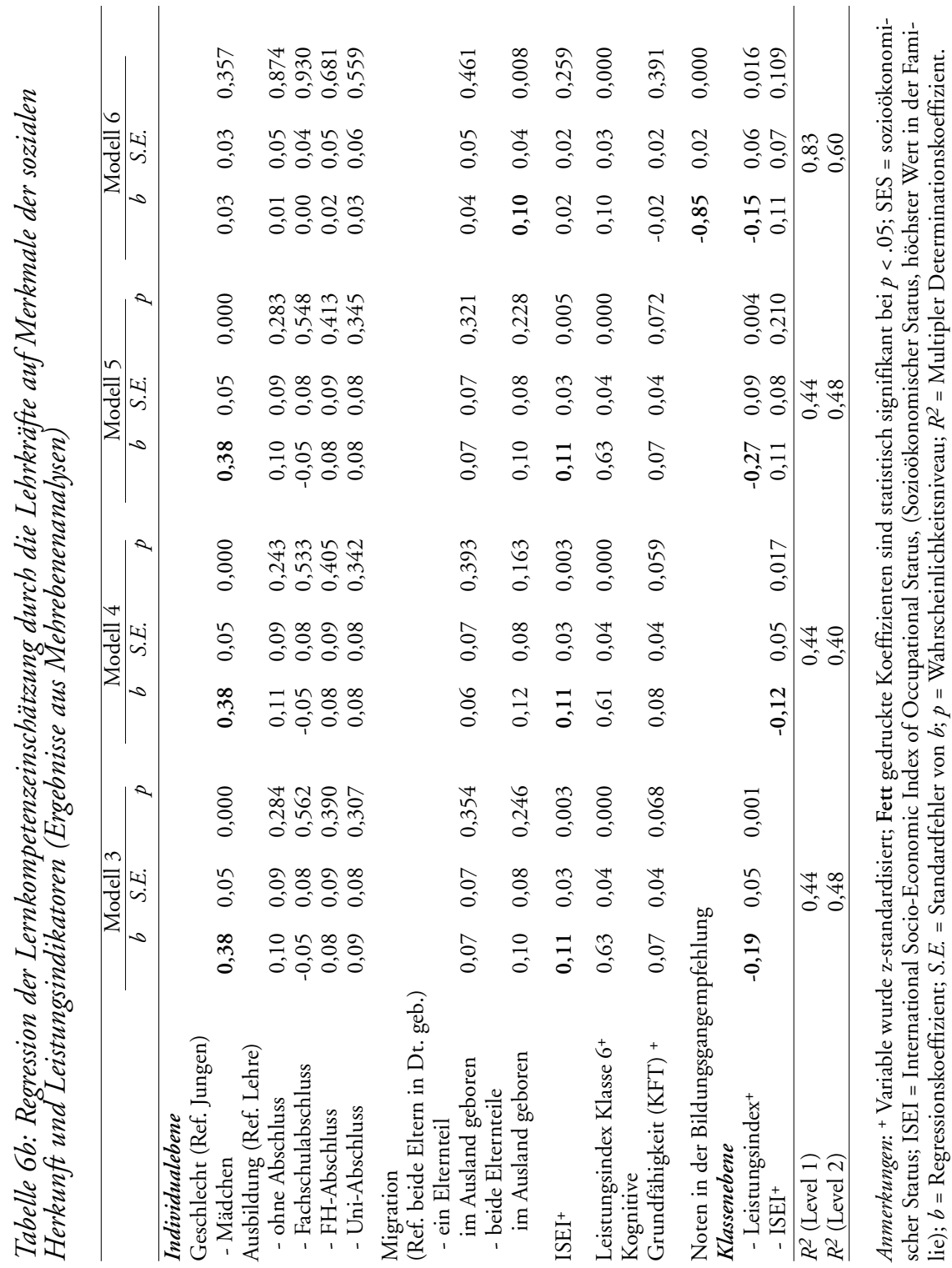

In der vorliegenden Studie stand der auf Klassenebene gemittelte ISEI-Wert in einem negativen Zusammenhang mit den Lernkompetenzeinschätzungen (Modell 4). Demnach fiel die Lernkompetenzeinschätzung bei Kontrolle aller individuellen Schülermerkmale in Klassen, die eine günstige soziale Zusammensetzung aufweisen, weniger positiv aus. In der simultanen Analyse der leistungs- 
bezogenen und sozialen Komposition der Klasse (Modell 5) zeigte sich in Analogie zu den Befunden für die Noten nur noch für die mittlere Klassenleistung ein spezifischer Effekt auf die Lernkompetenzeinschätzungen. Dieser fand sich in abgeschwächter Form - auch dann, wenn auf der Individualebene die Noten kontrolliert wurden (Modell 6).

\section{Diskussion}

In der vorliegenden Studie haben wir uns am Beispiel eines Bundeslandes mit einer zentralen Facette des Übergangs von der Grundschule in die Sekundarstufe I auseinandergesetzt. Für die Bildungsgangempfehlung schätzen in Berlin die Lehrkräfte die Lernkompetenz aller Schülerinnen und Schüler ein; diesen Einschätzungen kommt dann eine besondere Rolle bei der Übertrittsempfehlung zu, wenn die Notendurchschnitte keine direkte Schulformempfehlung zulassen.

Es konnte gezeigt werden, dass die Regeln der Übergangsverordnung bei Vorliegen der explizit benannten Notenintervalle in fast allen Fällen auch umgesetzt wurden. In ca. 37 Prozent aller Fälle, deren Noten ausserhalb dieser Intervalle lagen, war jedoch keine direkt aus dem Notendurchschnitt ableitbare Empfehlung möglich. In den meisten derartiger Fälle gaben die Lehrkräfte eine Empfehlung für den weniger anspruchsvolleren Bildungsgang ab.

Verschiedene Studien haben einen positiven Effekt des sozioökonomischen Status auf die Notenvergabe nachgewiesen. Schülerinnen und Schüler aus sozial begünstigten Familien bekommen bei gleicher Leistung, gemessen mit einem standardisierten Leistungstest, bessere Noten als Kinder aus sozial weniger begünstigten Familien. Dennoch folgt hieraus nicht zwangsläufig eine Benachteiligung von Schülerinnen und Schülern aus Familien mit niedrigem sozialem Hintergrund hin, denn zu bedenken ist, dass Schulnoten mehr abdecken und mehr abdecken sollen als nur die «objektive» Leistung einer Schülerin bzw. eines Schülers, wie sie mit einem schriftlichen Test gemessen wird. Die Zensurenvergabe unterliegt ministeriell festgelegten Regularien, deren konkrete Ausgestaltung u.a. vom Fach, der Schulart und der Schulstufe abhängt. In jedem Fall jedoch gilt, dass sich Noten aus verschiedenen Einzelleistungen (schriftliche und mündliche Leistungen, Hausaufgaben) zusammensetzen. Daher ist nicht zu erwarten, dass mit einem standardisierten Leistungstest Noten perfekt vorausgesagt werden können. Wäre dies der Fall, würden die Noten ihren Zweck verfehlt haben. Der Anteil der mündlichen Leistung oder der Hausaufgaben wird somit in den Modellen nicht berücksichtigt, was zu einer Unterspezifikation des Modells (und damit zu einer unzureichenden Kontrolle von Drittvariablen) führt. Damit besteht ein Risiko, den Einfluss der sozialen Herkunft auf die Notenvergabe zu überschätzen.

Der Effekt der sozialen Herkunft könnte genau an der Stelle wirken, die nicht durch den Test abgedeckt wird. Unterschiede in der Benotung, auch wenn die 
Vergaberegeln korrekt angewendet werden, sind nämlich möglicherweise eher auf primäre Effekte in den Herkunftsfamilien (z.B. Sozialisation oder elterliche Unterstützung) zurückzuführen, die sich in besonderer Beteiligung der Schülerinnen und Schüler am Unterricht und in sorgfältig ausgeführten Hausaufgaben ausdrücken, als auf Lehrereffekte. Würde man testen wollen, ob der soziale Hintergrund sich in unangemessener Weise in den Noten widerspiegelt, müsste man demnach alle relevanten Komponenten bei der Notenvergabe berücksichtigen. Der Effekt der sozialen Herkunft sollte dann keine prädiktive Wirkung mehr auf die Notenvergabe nehmen (bzw. sich deutlich reduzieren), wenn diese zusätzlichen Prädiktoren berücksichtigt wurden. Auf diese Weise könnte die Diskriminierungsthese, nach der die Schule Kinder aus sozial weniger begünstigten Familien bei der Notenvergabe systematisch benachteiligt, widerlegt - oder bestätigt - werden.

Schulnoten kommt bei der Vergabe von Grundschulempfehlungen eine zentrale Rolle zu. Fliessen Merkmale des sozialen Hintergrunds in die Benotung ein, so hat dies unmittelbare Bedeutung für die Grundschulempfehlung. Sofern zusätzlich zu den Noten weitere Merkmale, wie die Einschätzung der Lernkompetenz, in die Empfehlungsvergabe einfliessen, stellt sich die Frage, ob sich vergleichbare Effekte der sozialen Herkunft nachweisen lassen. In Einklang mit unseren Erwartungen ergab sich ein positiver prädiktiver Effekt des sozioökonomischen Status auf die Schulnoten und die Einschätzung der Lernkompetenz. Bei gleichen Leistungen in den standardisierten Leistungstests erhielten Schülerinnen und Schüler mit günstigem sozioökonomischem Hintergrund vergleichsweise bessere Einschätzungen als Schülerinnen und Schüler mit weniger günstigem sozioökonomischem Hintergrund. Handelt es sich dabei um ungerechte Beurteilungen oder gar um "Diskriminierungseffekte», da ja der tatsächliche Leis tungsstand mithilfe eines standardisierten Leistungstests kontrolliert wurde? In der Tat kann auf der Basis der vorliegenden Daten nicht ausgeschlossen werden, dass Lehrkräfte bewusst oder unbewusst ihre Schulformempfehlung (auch) an dem sozialen Hintergrund der Schülerinnen und Schüler orientieren, beispielsweise weil sie vermuten, dass ein günstiger sozioökonomischer Hintergrund eine Ressource beim Gymnasialbesuch darstellt. Lehrkräfte könnten also die grösseren finanziellen und fachlichen Ressourcen der Ober- und Mittelschichtfamilien mit in ihrem Entscheidungsprozess berücksichtigen (Arnold et al., 2007; Ditton, 2005). Allerdings ist es ebenso möglich, dass die unterschiedlichen Bewertungen in Form von Noten sowie Einschätzungen der Lernkompetenz zumindest teilweise tatsächlich zu beobachtende Unterschiede im Arbeits- und Lernverhalten der Schülerschaft ausdrücken, die sich in den Leistungstests nicht widerspiegeln. Der SES-Effekt wäre in diesem Falle ein Indikator für tatsächlich bestehende, von uns aber nicht erfasste Unterschiede bei der Schülerschaft, die systematisch mit der familiären Herkunft kovariieren. In diesem Falle würde es sich nicht um eine Benachteiligung handeln.

Entsprechend unseren Erwartungen zeigte sich, dass Mädchen bei den Lern- 
kompetenzeinschätzungen besser beurteilt werden als Jungen - auch dann, wenn die individuelle Leistung kontrolliert wird. Dieser Geschlechtereffekt liesse sich analog zum positiven SES-Effekt einerseits möglicherweise als eine Benachteiligung der Jungen durch die Lehrkräfte interpretieren. Demnach berücksichtigen die Lehrkräfte bei der Einschätzung der Lernkompetenz systematisch das Geschlecht der Schülerinnen und Schüler und schreiben den Mädchen bei gleicher individueller Testleistung eine grössere Lernkompetenz zu als den Jungen. Andererseits könnten in diesem Effekt aber auch tatsächliche Unterschiede im Arbeits- und Lernverhalten zwischen den Mädchen und Jungen zum Ausdruck kommen, sodass die berichteten Unterschiede tatsächlich vorhandene Unterschiede widerspiegeln würden.

Die Analyse von Referenzgruppeneffekten ergab einen signifikanten Effekt der auf Klassenebene gemittelten Testleistung auf die Lernkompetenzeinschätzung. Demnach fällt bei gleicher individueller Testleistung die Einschätzung in einer leistungsstarken Klasse weniger positiv aus. Für die soziale Zusammensetzung der Klasse konnte bei Kontrolle der Leistungszusammensetzung der Klasse kein zusätzlicher Effekt auf die Lernkompetenzeinschätzung nachgewiesen werden. Dasselbe Befundmuster der Klassenmerkmale zeigte sich auch bei den Schulnoten, wonach Schülerinnen und Schüler bei gleicher individueller Leistung in leistungsstarken Klassen strenger benotet werden.

Abschliessend stellt sich die Frage nach der praktischen Relevanz der vorliegenden Befunde. Sieht ein Bildungssystem unterschiedliche Schulformen oder Bildungsgänge vor, muss es auch Regelungen geben, wie die Allokation auf die verschiedenen Bildungsgänge erfolgen soll. Die Kritik an den Verfahren der Bildungsgangzuteilung ist leicht formuliert, konkrete Alternativen stehen jedoch bislang noch weitestgehend aus. Liesse man beispielsweise Eltern frei entscheiden, würde die soziale Selektivität des Übergangs in die Sekundarstufe I sicherlich grösser ausfallen (Ditton, 2005), denn Bildungsentscheidungen werden im Kontext der eigenen sozialen Stellung getroffen (Maaz, 2006) und die Kosten- und Nutzenabwägungen, die einer Entscheidung zugrunde liegen, variieren zwischen den Sozialschichten (Becker, 2007; Boudon, 1974). Es geht also um die konkrete Ausgestaltung der Übergangsregelungen. Grundlage der vorliegenden Studie ist die Berliner Regelung, die sich durch einen breiten Kranz von Indikatoren auszeichnet, unter anderem Noten, die Lernkompetenzeinschätzungen durch die Lehrkräfte und der Elternwille. Der wohl zentralste Befund der vorliegenden Studie sind die Referenzgruppeneffekte, denen Lehrkräfte bei der Einschätzung der Lernkompetenz ebenso wie bei der Notenvergabe unterliegen. Obwohl diese Einschätzungen möglicherweise zusätzlich zu den Noten eine prädiktive Validität in Hinblick auf die Leistungen in der Sekundarschule haben könnten (Baeriswyl et al., 2007; entsprechende Analysen mit dem ELEMENT-8-Datensatz sind geplant), scheinen sie nur bedingt dazu geeignet zu sein, die bekannten Referenzgruppeneffekte bei der Übertrittsentscheidung auszuschalten. 
Auch wenn die vorliegende Studie Forschungslücken schliesst, so ist noch einer Reihe von Aspekten nachzugehen. Hierzu gehört die theoretische wie empirische Aufarbeitung des Konstrukts Lernkompetenz und des Prozesses, wie Lehrkräfte zu Urteilen gelangen. Hierzu gehört ebenfalls der Einbezug von Faktoren jenseits von Schule. Die angemessene Untersuchung dieser Fragen stell hohe methodische Anforderungen, die erfüllt werden müssen, zumal die gesamte Thematik bei hoher praktischer Relevanz in einem bildungspolitisch umstrittenen Gebiet angesiedelt ist.

\section{Anmerkungen}

1 In der vorliegenden Studie wird die Bezeichnung Bildungsgangempfehlung für die gesamte Einschätzung der Schülerinnen und Schüler durch die Lehrkräfte am Ende der Grundschulzeit herangezogen. Hierzu zählen neben der Dokumentation der Schulnoten auch Lernkompetenzeinschätzungen der Lehrkräfte. In Berlin werden diese Informationen und Einschätzungen zum Halbjahr der sechsten Klassenstufe in einer Bildungsgangempfehlung dokumentiert, in der schliesslich eine Empfehlung für einen Bildungsgang in der Sekundarstufe abgegeben wird. Wenn von Grundschulempfehlung oder der Empfehlung für eine der weiterführenden Schulformen bzw. Bildungsgänge die Rede ist, ist damit die konkrete Empfehlung für die Hauptschule, Realschule oder das Gymnasium gemeint.

2 Mit der Durchschnittsnote ist im Folgenden der in der Bildungsgangempfehlung dokumentierte Notendurchschnitt gemeint.

3 Um die faktorielle Struktur der Einschätzungen zu prüfen, wurden eine Reihe von konfirmatorischen Faktorenanalysen mit dem Programm Mplus (Muthén \& Muthén, 19982008) gerechnet. Ein sogenanntes Nested-Faktor-Modell, bei dem die Ausprägungen auf den einzelnen Items durch einen generellen Faktor sowie durch drei spezifische Faktoren (Arbeitsprinzipien, Regulation, Arbeitstechniken) erklärt werden, wies hierbei den besten Fit auf $\left(\chi^{2}=518,43, \mathrm{df}=75, \mathrm{CFI}=0,972\right.$, RMSEA $\left.=0,078\right)$. Dies legt den Schluss nahe, dass die post hoc von uns formulierten Faktoren durch einen Generalfaktor überlagert werden.

\section{Literatur}

Allison, P. D. (2001). Missing data. Thousand Oaks, CA: Sage.

Arnold, K.-H., Bos, W., Richert, P. \& Stubbe, T. C. (2007). Schulaufbahnpräferenzen am Ende der vierten Klassenstufe. In W. Bos et al. (Hrsg.), IGLU 2006. Lesekompetenzen von Grundschulkindern in Deutschland im internationalen Vergleich (S. 271-297). Münster: Waxmann.

Bacher, J. (2003). Bildungsungleichheit und Bildungsbenachteiligung im weiterführenden Schulsystem Österreichs. Eine Sekundäranalyse der PISA 2000-Erhebung. SWS-Rundschau, 45, 37-62.

Bacher, J. (2005). Soziale Ungleichheit und Bildungspartizipation im weiterführenden Schulsystem Österreichs. Österreicherische Zeitschrift für Soziologie, 28, 3-32.

Baeriswyl, F., Trautwein, U., Wandeler, C. \& Lüdtke, O. (2007). Testleistung, Noten, Übertrittsempfehlung oder Übertrittsgutachten: Vorhersagekraft unterschiedlicher Elemente von Übertrittsverfahren am Ende der Grundschule für die Schulleistung am Ende der Sekundarstufe I. Manuskript zur Publikation eingereicht.

Baeriswyl, F., Wandeler, C., Trautwein, U. \& Oswald, K. (2006). Leistungstest, Offenheit von Bildungsgängen und obligatorische Beratung der Eltern: Reduziert das Deutschfreiburger Übergangsmodell die Effekte des sozialen Hintergrunds bei Übergangsentscheidungen? Zeitschrift für Erziehungswissenschaft, 9, 373-392. 
Baumert, J. \& Schümer, G. (2001). Familiäre Lebensverhältnisse. Bildungsbeteiligung und Kompetenzerwerb. In J. Baumert, E. Klieme, M. Neubrand, M. Prenzel, U. Schiefele, W. Schneider, P. Stanat, K.-J. Tillmann \& M. Weiss (Hrsg.), PISA 2000. Basiskompetenzen von Schülerinnen und Schülern im internationalen Vergleich (S. 323-407). Opladen: Leske + Budrich.

Baumert, J., Trautwein, U. \& Artelt, C. (2003). Schulumwelten - institutionelle Bedingungen des Lehrens und Lernens. In J. Baumert, C. Artelt, E. Klieme, M. Neubrand, M. Prenzel, U. Schiefele, W. Schneider, K.-J. Tillmann \& M. Weiss (Hrsg.), PISA 2000. Ein differenzierter Blick auf die Länder der Bundesrepublik Deutschland (S. 261-331). Opladen: Leske + Budrich.

Becker, R. (2007). Soziale Ungleichheit von Bildungschancen und Chancengleichheit. In R. Becker \& W. Lauterbach (Hrsg.), Bildung als Privileg? Erklärungen und Befunde zu den Ursachen der Bildungsungleichheit (S. 157-185). Wiesbaden: VS Verlag für Sozialwissenschaften.

Bliese, P. D. (2000). Within-group agreement, non-independence, and reliability: Implications for data aggregation and analysis. In K. J. Klein \& S. W. Kozlowski (Eds.), Multilevel theory, research, and methods in organizations (pp. 349-381). San Francisco, CA: JosseyBass.

Bos, W., Lankes, E.-M., Prenzel, M., Schwippert, K., Walther, G. \& Valtin, R. (Hrsg.). (2003). Erste Ergebnisse aus IGLU. Schülerleistungen am Ende der vierten Jahrgangsstufe im internationalen Vergleich. Münster: Waxmann.

Bos, W., Voss, A., Lankes, E.-M., Schwippert, K., Thiel, O. \& Valtin, R. (2004). Schullaufbahnempfehlungen von Lehrkräften für Kinder am Ende der vierten Jahrgangsstufe. In W. Bos, E.-M. Lankes, M. Prenzel, K. Schwippert, R. Valtin \& G. Walther (Hrsg.), IGLU. Einige Länder der Bundesrepublik Deutschland im nationalen und internationalen Vergleich (S. 191-220). Münster: Waxmann.

Boudon, R. (1974). Education, opportunity, and social inequality: Changing prospects in Western society. New York: Wiley.

Ditton, H. (2005). Der Beitrag von Familie und Schule zur Reproduktion von Bildungsungleichheit. In H. G. Holtappels \& K. Höhmann (Hrsg.), Schulentwicklung und Schulwirksamkeit. Systemsteuerung, Bildungschancen und Entwicklung der Schule. 30 Jahre Institut für Schulentwicklungsforschung (S. 121-130). Weinheim: Juventa.

Ditton, H. (2007). Schulübertritte, Geschlecht und soziale Herkunft. In H. Ditton (Hrsg.), Kompetenzaufbau und Laufbahnen im Schulsystem. Ergebnisse einer Längsschnittuntersuchung an Grundschulen (S. 63-87). Münster: Waxmann.

Ditton, H. \& Krüsken, J. (2006): Sozialer Kontext und schulische Leistungen - zur Bildungsrelevanz segregierter Armut. Zeitschrift für Soziologie der Erziehung und Sozialisation, 26, $135-157$.

Ganzeboom, H. B. G., DeGraaf, P. M., Treiman, D. J. \& DeLeeuw, J. (1992). A standard international socio-economic Index of occupational status. Social Science Research, 21, 1-56.

Grundschulverordnung. (2005). Verordnung über den Bildungsgang der Grundschule. Vom 19. Januar 2005, zuletzt geändert durch Verordnung vom 11. Dezember 2007. Berlin: Senatsverwaltung für Bildung, Wissenschaft und Forschung.

Heinzel, F. \& Prengel, A. (Hrsg.). (2002). Heterogenität, Integration und Differenzierung in der Primarstufe. Jahrbuch Grundschulforschung 6. Opladen: Leske + Budrich.

Heller, K. A. \& Perleth, C. (2000). Kognitiver Fähigkeitstest für 4. bis 12. Klassen, Revision. Göttingen: Beltz.

Ingenkamp, K. (1993). Der Prognosewert von Zensuren, Lehrergutachten, Aufnahmeprüfungen und Tests während der Grundschulzeit für den Sekundarschulerfolg. In R. Olechowski \& E. Persy (Hrsg.), Frühe schulische Auslese (S. 68-85). Frankfurt/Main: Peter Lang.

Ingenkamp, K. \& Lissmann, U. (2005). Lehrbuch der Pädagogischen Diagnostik. Weinheim: Beltz. 
KMK = Sekretariat der Ständigen Konferenz der Kultusminister der Länder in der Bundesrepublik Deutschland (2006). Übergang von der Grundschule in Schulen des Sekundarbereichs I. Informationsunterlage des Sekretariats der Kultusministerkonferenz, Stand: März 2006.

Lehmann, R., Gänsfuss, R. \& Peek, R. (1999). Aspekte der Lernausgangslage und der Lernentwicklung von Schülerinnen und Schülern an Hamburger Schulen - Klassenstufe 7. Hamburg: Behörde für Schule, Jugend und Berufsbildung, Amt für Schule.

Lehmann, R. \& Lenkeit, J. (2008). ELEMENT. Erhebung zum Lese- und Mathematikverständnis. Entwicklungen in den Jahrgangsstufen 4 bis 6 in Berlin. Abschlussbericht über die Untersuchungen 2003, 2004 und 2005 an Berliner Grundschulen und grundständigen Gymnasien. Berlin: Humboldt Universität.

Lehmann, R. H., Peek, R., Gänsfuss, R., Lutkat, S., Mücke, S. \& Barth, I. (2001). QuaSUM. Qualitätsuntersuchung an Schulen zum Unterricht in Mathematik. Ergebnisse einer repräsentativen Untersuchung im Land Brandenburg. Potsdam: Ministerium für Bildung, Jugend und Sport des Landes Brandenburg.

Lüdtke, O., Robitzsch, A., Trautwein, U. \& Köller, O. (2007). Umgang mit fehlenden Werten in der psychologischen Forschung: Probleme und Lösungen. Psychologische Rundschau, 58, 103-117.

Lüdtke, O., Trautwein, U., Kunter, M. \& Baumert, J. (2006). Analyse von Lernumwelten. Ansätze zur Bestimmung der Reliabilität und Übereinstimmung von Schülerwahrnehmungen. Zeitschrift für Pädagogische Psychologie, 20, 85-96.

Maaz, K. (2006). Soziale Herkunft und Hochschulzugang. Effekte institutioneller Öffnung im Bildungssystem. Wiesbaden: VS Verlag für Sozialwissenschaften.

Maaz, K., Hausen, C., McElvany, N. \& Baumert, J. (2006). Stichwort: Übergänge im Bildungssystem. Theoretische Konzepte und ihre Anwendung in der empirischen Forschung beim Übergang in die Sekundarstufe. Zeitschrift für Erziehungswissenschaft, 9, 299-327.

Maaz, K., Neumann, M. \& Trautwein, U. (in Druck). Schulsysteme im deutschsprachigen Raum. In W. Sacher, L. Haag, T. Bohl, G. Lang-Wojtasik \& S. Blömeke (Hrsg.), Handbuch Schule: Theorie, Geschichte, Organisation, Entwicklung, Evaluation. Bad Heilbrunn: Klinkhardt.

Marsh, H. W. (1987). The big-fish-little-pond effect on academic self-concept. Journal of Educational Psychology, 79, 280-295.

Marsh, H. W. (2005). The big-fish-little-pond effect on academic self-concept. Zeitschrift für Pädagogische Psychologie, 19, 119-128.

Merkens, H. \& Wessel, A. (2002). Zur Genese von Bildungsentscheidungen. Eine empirische Studie in Berlin und Brandenburg. Baltmannsweiler: Schneider Verlag Hohengehren.

Moser, U. \& Rhyn, H. (2000). Lernerfolg in der Primarschule. Aarau: Sauerländer.

Muthén, B. O. \& Muthén, L. K. (1998-2008). Mplus (Version 5.1) [Computer Software]. Los Angeles, CA: Muthén \& Muthén.

Ramseier, E. \& Brühwiler, C. (2003). Herkunft, Leistung und Bildungschancen im gegliederten Bildungssystem: Vertiefte PISA-Analysen unter Einbezug der kognitiven Grundfähigkeiten. Schweizerische Zeitschrift für Bildungswissenschaften, 25, 1-34.

Raudenbush, S. W. \& Bryk, A. S. (2002). Hierarchical linear models (2nd ed.). Thousand Oaks, CA: Sage.

Raudenbush, S. W., Bryk, A. S., Cheong, Y. \& Congdon, R. T. (2004). HLM 6: Hierarchical linear modeling. Chicago: Scientific Software International.

Rösner, E. (2007). Hauptschule am Ende. Ein Nachruf. Münster: Waxmann.

Rubin, D. B. (1987). Multiple imputation for nonresponse in surveys. New York: Wiley.

Schafer, J. L. (1999). NORM: Multiple imputation of incomplete multivariate data under a normal model, version 2. Software for Windows 95/98/NT, available from http://www.stat.psu.edu/ - jls/misoftwa.html.

Schafer, J. L. \& Graham, J. W. (2002). Missing data: Our view of the state of the art. Psychological Methods, 7, 147-177. 
Snijders, T. A. B. \& Bosker, R. J. (1999). Multilevel analysis: An introduction to basic and advanced multilevel modeling. London: Sage.

Thiel, O. (2005). Modellierung der Bildungsgangempfehlung in Berlin. Unveröffentlichte Dissertation, Humboldt-Universität zu Berlin.

Tiedemann, J. \& Billmann-Mahecha, E. (2007). Zum Einfluss von Migration und Schulklassenzugehörigkeit auf die Übergangsempfehlung für die Sekundarstufe I. Zeitschrift für Erziehungswissenschaft, 10, 108-120.

Trautwein, U. \& Baeriswyl, F. (2007). Wenn leistungsstarke Klassenkameraden ein Nachteil sind: Referenzgruppeneffekte bei Übergangsentscheidungen. Zeitschrift für Pädagogische Psychologie, 21, 119-133.

Trautwein, U., Gerlach, E. \& Lüdtke, O. (in press). The detrimental effects of athletic classmates: A longitudinal study on frame of reference effects. Journal of Educational Psychology.

Trautwein, U., Lüdtke, O., Marsh, H. W., Köller, O. \& Baumert, J. (2006). Tracking, grading, and student motivation: Using group composition and status to predict self-concept and interest in ninth-grade mathematics. Journal of Educational Psychology, 98, 788-806.

Warm T. A. (1989). "Weighted likelihood estimation of ability in item response theory». Psychometrika, 54, 427-450.

Schlagworte: sozialer Hintergrund, Referenzgruppeneffekt, Übergangsempfehlung, Lehrerurteil, Lernkompetenz, Grundschule

\section{La transition entre l'école primaire et l'école secondaire : le rôle des caractéristiques des élèves et des classes dans l'évaluation des compétences d'apprentissage individuelles par les enseignants}

\section{Résumé}

Le passage aux différentes filières du degré secondaire est une transition centrale dans la vie des jeunes. Cet article étudie la question de l'évaluation des compétences d'apprentissage individuelles par les enseignants à la fin de l'école primaire.

En sus des effets prédictibles inférés des caractéristiques des performances individuelles et des données socio-économiques présentes, nous analysons si les estimations que font les enseignants à propos des compétences d'apprentissage individuelles des élèves sont systématiquement reliées aux performances moyennes de la classe. L'analyse empirique a été conduite sur la base d'un échantillon de 976 élèves berlinois à la fin de l'école primaire.

Les analyses multiniveaux montrent, comme attendu, que les indicateurs de performances tout comme le statut socio-économique des élèves sont positivement associés aux estimations de compétences d'apprentissage. Par ailleurs, le contrôle des performances individuelles fait apparaître un coefficient de régression négatif relatif aux niveaux moyens de performance sur l'estimation des capacités d'apprentissage. Ceci peut être interprété comme un effet du groupe de référence. 
En outre, un coefficient de régression négatif se retrouve auprès du contrôle des performances individuelles du niveau moyen des performances de la classe sur les estimations de compétences à l'apprentissage, ce qui peut être interprété en tant qu'effet du groupe de référence.

Mots clés: contexte social, effet du groupe de référence, recommandations transitoires, jugement professionnel, compétences d'apprentissages, école primaire

\section{La transizione verso le scuole secondarie: il ruolo delle caratteristiche degli allievi e della classe nella valutazione delle competenze da parte degli insegnanti}

\section{Riassunto}

Il passaggio verso i diversi percorsi della formazione secondaria è uno dei momenti chiave nella vita dei giovani. Questo contributo analizza la valutazione delle competenze degli allievi alla fine della scuola di base. Accanto al valore predittivo delle caratteristiche individuali e del contesto socio-economico, si è analizzata la relazione tra giudizio degli insegnanti e livello di prestazione medio della classe. L'analisi empirica si basa su un campione di 976 allievi alla fine della scuola di base a Berlino. Dai risultati emerge una nesso positivo tra il giudizio degli insegnanti da un lato e gli indicatori individuali e socioeconomici dall'altro lato. Il controllo delle prestazioni individuali ha poi portato alla constatazione di un coefficiente di regressione negativo del livello di prestazione medio della classe sul giudizio, il che può essere interpretato come un effetto del gruppo di referenza.

Parole chiave: contesto sociale, giudizio degli insegnanti, competenza, effetto del gruppo di riferimento, scuola di base

\section{Teacher Assessments of Individual Student Competence at the Transition to Secondary Education: The Role of Student and Class Characteristics}

\footnotetext{
Abstract

The transition to the different tracks of the secondary system is one of the major changes of status in young people's lives. This article examines teachers' assessments of individual student competence at the end of elementary education, based on a sample of 976 students approaching the end of elementary schooling in Berlin. In addition to the predictive effects of individual achievement characteristics and social background indicators, the study examined whether teachers' assessments of student competence were systematically related to class-mean achievement. Multilevel analyses showed that, as expected, achievement indica-
} 
tors and socioeconomic status were positively associated with teachers' assessments of student competence. Moreover, when individual achievement was controlled, the regression coefficient of class-mean achievement on teachers' assessments of student competence was negative. This finding can be interpreted as a reference group effect.

Key words: social background, Big-Fish-Little-Pond-Effekt, transition, assessment, learning competence, elementary school, 